\title{
Critique of the fuzzball program
}

\author{
Suvrat Raju* and Pushkal Shrivastava ${ }^{\dagger}$ \\ International Centre for Theoretical Sciences, Tata Institute of Fundamental Research, \\ Shivakote, Bengaluru 560089, India
}

(Received 8 October 2018; published 25 March 2019)

\begin{abstract}
We explore the viability of fuzzballs as candidate microstate geometries for the black hole and their possible role in resolutions of the information paradox. We argue that if fuzzballs provide a description of black-hole microstates then the typical fuzzball geometry can only differ significantly from the conventional black-hole geometry at a Planck-scale distance from the horizon. However, precisely in this region, quantum fluctuations in the fuzzball geometry become large, and the fuzzball geometry becomes unreliable. We verify these expectations through a detailed calculation of quantum expectation values and quantum fluctuations in the two-charge fuzzball geometries. We then examine some of the solutions discovered in the work by Bena et al. [Phys. Rev. Lett. 117, 201601 (2016)]. We show, based on a calculation of a probe two-point function in this background, that these solutions, and others in their class, violate robust expectations about the gap in energies between successive energy eigenstates and differ too much from the conventional black hole to represent viable microstates. We conclude that, while fuzzballs are interesting starlike solutions in string theory, they do not appear to be relevant for resolving the information paradox and cannot be used to make valid inferences about black-hole interiors.
\end{abstract}

DOI: 10.1103/PhysRevD.99.066009

\section{INTRODUCTION}

In higher-dimensional supergravities, it is sometimes possible to find horizonless solutions, called fuzzballs, with the same charges as a black hole. Over the past few years, considerable effort has been devoted to discovering and analyzing new classes of fuzzball solutions. Such solutions can be found in both asymptotically flat space and asymptotically anti-de Sitter space.

The fuzzball program (see Refs. [1,2] and references therein) is the bold idea that such geometries can be used to parametrize the set of microstates in quantum gravity that correspond to a black hole. The fuzzball program suggests that the black hole should be viewed only as some kind of "average" geometry, with individual microstates specified by distinct horizonless geometries. This has two immediate implications: first, that the entropy of the black hole can be recovered by quantizing the moduli space of fuzzball solutions and, second, that the black hole truly has no interior.

\footnotetext{
suvrat@icts.res.in

pushkal.shrivastava@icts.res.in
}

Published by the American Physical Society under the terms of the Creative Commons Attribution 4.0 International license. Further distribution of this work must maintain attribution to the author(s) and the published article's title, journal citation, and DOI. Funded by SCOAP ${ }^{3}$.
The fuzzball program has not been carried through to completion in any setting corresponding to a macroscopic black hole. The moduli space of fuzzballs has only been quantized [3] for fuzzballs that are dual to ground states of the D1-D5 system [4,5], which do not correspond to a black hole with a macroscopic horizon. Nevertheless, given the considerable effort that continues to be directed toward understanding fuzzballs, we believe it is pertinent to address the following question: is it consistent with the principles of statistical mechanics to expect that black-hole microstates can be represented by distinct geometries, which can be analyzed by studying classical solutions?

In essence, our analysis is very simple. First, we point out that quantum-mechanical microstates in a system with $e^{S}$ states are almost all indistinguishable from one another; when microstates are probed with any reasonable observable, the differences between distinct typical states are of size $e^{-\frac{S}{2}}$. Now, quantum fluctuations of the metric about each microstate are suppressed by a power of $S$, and therefore the differences between typical microstates are much smaller than the quantum fluctuations of the geometry about each microstate. Therefore, it seems clear to us that one should not attempt to capture these differences at the level of the geometry but rather ascribe the same geometry to all of them. This implies that microstates that occupy almost all of the volume of the Hilbert space must be represented by a single universal geometry. We further argue below that this must be the conventional black-hole geometry. 
Then, we consider the weaker possibility that, while fuzzballs might not provide a useful representation of typical states, they might still provide a basis that spans all black-hole microstates. In this context, we prove some simple bounds on how atypical basis elements can be. We use these bounds to show that if fuzzballs are to form even only a basis then, in most of space, typical fuzzball metrics must equal the conventional black-hole metric up to terms that are suppressed by $\frac{1}{\sqrt{S}}$. So, in most regions of space, such fuzzballs are effectively indistinguishable from the black hole.

We further argue that if typical fuzzballs are to deviate significantly from the conventional black hole they can only do so once we are within Planck length of the horizon. We show that this deviation cannot occur at a larger scale like the string scale. Therefore, if fuzzballs are to be microstates, then typical fuzzballs must be represented by metrics with explicit Planck-scale structures. We argue that such metrics cannot be analyzed classically-either in supergravity or in classical string theory-since quantum fluctuations in such metrics are of the same order as the classical Planck-scale structures. Therefore, in the region where fuzzballs may have displayed interesting deviations from the black hole, they are unreliable.

In fact, several of the explicit fuzzball solutions that have been found have structures at macroscopic scales-much larger than the Planck scale. (In the terminology of Ref. [2], such solutions are called "microstate geometries.") We argue that such fuzzballs are irrelevant to the discussion of black-hole microstates since they do not satisfy our bounds on how close elements of a basis have to be to the ensemble average.

The arguments above are explained in greater detail in Sec. II. It is sometimes argued that fuzzballs are required to resolve the information paradox [6-8], and we explain, in Sec. II C, why we believe that this argument is incorrect.

We believe that our arguments are robust, but in order to check these arguments, we performed several detailed calculations with explicit examples of fuzzball solutions. These calculations, which are described in Secs. III and IV, take up the bulk of this paper; the reader who is not persuaded by our abstract arguments in Sec. II should consult these concrete examples.

In Sec. III, we analyze the Lunin-Mathur geometries corresponding to ground states in the D1-D5 system [5,9] that were quantized by Rychkov [3]. In this quantumstatistical system, we are able to verify our general expectations. We can compute both the quantum-mechanical expectation of components of the metric and also fluctuations in these quantities. We show that the geometry corresponding to a typical microstate differs from the conventional solution only at the Planck scale. Moreover, in the region where the geometry differs in an interesting manner, the fluctuations in physical quantities become the same size as their classical expectation values, and the geometry becomes unreliable, precisely in line with our general expectations.

In Sec. IV, we analyze the recently discovered class of asymptotically anti-de Sitter (AdS) solutions that correspond to 1/4-BPS states in the D1-D5 system [10]. Such 1/4-BPS states are described by a black hole with finite horizon area [11], but the geometries of Ref. [10] differ macroscopically from the black-hole geometry. We show that these differences can be easily detected through simple asymptotic boundary observables.

More specifically, in these geometries, we compute the two-point Wightman function and commutator of a marginal boundary scalar operator. We show that the support of these functions in frequency space is concentrated on a set of discrete well-separated frequencies, in contradiction to

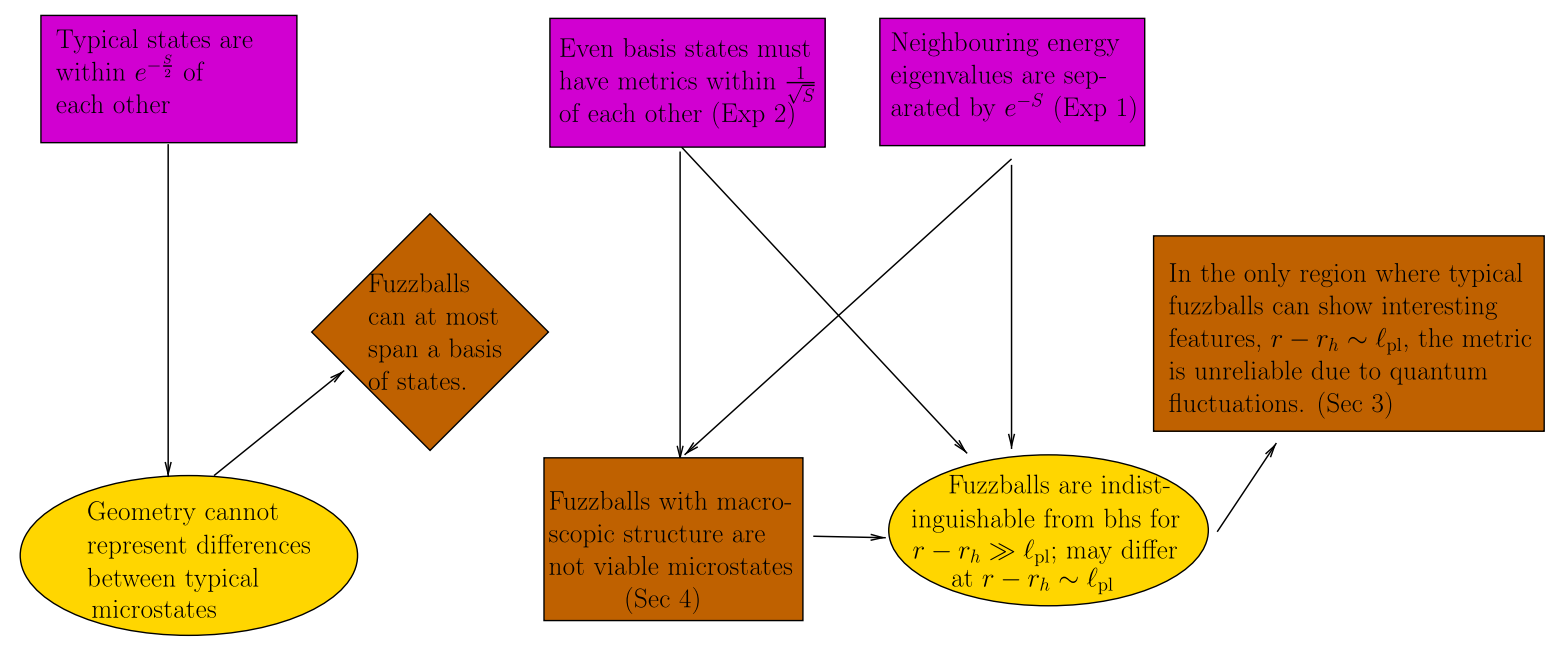

FIG. 1. Logical flowchart of the paper. Statistical mechanics results are in magenta rectangles. The physical expectation on which they rely is referred to in brackets. Implications for the fuzzball are in orange ovals. Major conclusions are in brown boxes. Sections in which a conclusion is verified are given in brackets. 
what one would expect for a black-hole microstate-for which we expect the support to be effectively continuous in frequency space. Next, we show that the falloff of these functions for large spatial momenta in the fuzzball geometry fails to saturate a bound that is saturated by the blackhole geometry. These calculations provide strong evidence that the geometries of Ref. [10] are not viable as typical microstates.

While our calculations in Sec. IV are specific to a class of solutions, we believe that our conclusions are far more general. If a geometry has macroscopic features, these macroscopic features can be detected by appropriate asymptotic correlation functions, and they lead to violations on the bounds of how atypical a basis for black-hole microstates can be.

Figure 1 outlines the flow of logic in this paper and explains how our calculations in Secs. III and IV fit into this flow.

\section{STATISTICAL-MECHANICS EVALUATION OF THE FUZZBALL PROGRAM}

In this section, we review some simple results from quantum statistical mechanics and explain their implications for the fuzzball program. These results will motivate our calculations in Secs. III and IV. We have organized this section into three subsections: in Sec. II A, we review some results from statistical mechanics; in Sec. II B, we explain the relevance of these results for the fuzzball program; and in Sec. II C, we discuss the "Hawking theorem" described in Ref. [7], which is sometimes used to indirectly infer properties of fuzzballs.

Some readers may be concerned that our arguments in this section are too abstract. We urge these readers to read this section in conjunction with Secs. III and IV, in which we have performed a number of calculations that support our deductions in specific examples.

\section{A. Some results from statistical mechanics}

We now discuss some results that characterize (a) typical states in high-dimensional quantum statistical systems, (b) the extent to which elements of a complete basis can differ from one another, and (c) the gap between neighboring energy eigenstates.

Result 1: Consider any subspace $H_{E}$ of a Hilbert space. Let $\operatorname{dim}\left(H_{E}\right)=e^{S}$, and let $\mu_{\psi}$ be the Haar measure on $H_{E}$ in the neighborhood of a state $|\psi\rangle$. Then, typical pure states in $H_{E}$ are exponentially close to the maximally mixed state on $H_{E}$ in the sense that for any Hermitian operator $A$ we have

$$
\langle A\rangle \equiv \int\langle\Psi|A| \Psi\rangle d \mu_{\psi}=\operatorname{Tr}(\rho A),
$$

where the density matrix $\rho=e^{-S} P$ and $P$ is the projector onto $H_{E}$. Moreover, deviations from this mean value are exponentially suppressed,

$$
\int(\langle\Psi|A| \Psi\rangle-\langle A\rangle)^{2} d \mu_{\psi} \leq \frac{\sigma_{\text {ens }}^{2}}{e^{S}+1},
$$

where $\sigma_{\text {ens }}^{2} \equiv \operatorname{Tr}\left(\rho A^{2}\right)-[\operatorname{Tr}(\rho A)]^{2}$.

To our knowledge, this result was first described in Ref. [12]. To prove this result, we choose some basis for the subspace, and we label its elements by $\left|f_{1}\right\rangle,\left|f_{2}\right\rangle \ldots\left|f_{e^{s}}\right\rangle$. Then, an arbitrary state in this subspace can be written as $|\Psi\rangle=\sum_{i} a_{i}\left|f_{i}\right\rangle$. The Haar measure is given by

$$
d \mu_{\Psi}=\frac{1}{V} \delta\left(\sum_{i=1}^{e^{S}}\left|a_{i}\right|^{2}-1\right) \prod_{j=1}^{e^{S}} d a_{j},
$$

where $V$ is a normalization constant, which can be set by demanding that $\int d \mu_{\Psi}=1$, which leads to $V^{-1}=\frac{\pi^{e^{S}}}{\Gamma\left(e^{S}\right)}$. We emphasize that the measure (2.1) is independent of the choice of basis.

Now, consider an arbitrary Hermitian operator, $A$, and denote its matrix elements in the basis above by $A_{i j}=\left\langle f_{j}|A| f_{i}\right\rangle$. Then,

$$
\begin{aligned}
\int\langle\Psi|A| \Psi\rangle d \mu_{\psi} & =\int d \mu_{\psi}\left[\sum_{i=1}^{e^{S}}\left|a_{i}\right|^{2} A_{i i}+\sum_{i \neq j} a_{i} a_{j}^{*} A_{i j}\right] \\
& =\frac{1}{e^{S}} \sum A_{i i}=\operatorname{Tr}(\rho A),
\end{aligned}
$$

where we have used the fact that $\int d \mu_{\psi} a_{i} a_{j}^{*}=\frac{1}{e^{S}} \delta_{i j}$.

A simple computation yields the variance in the second part of the result.

$$
\begin{aligned}
\int & {[\langle\Psi|A| \Psi\rangle-\operatorname{Tr}(\rho A)]^{2} d \mu_{\psi} } \\
& =\int\left[\sum_{i, j} A_{i j} a_{i} a_{j}^{*}-\sum_{i} A_{i i}\left|a_{i}\right|^{2}\right]^{2} \\
& =\int\left[\sum_{i \neq j, l \neq m} A_{i j} A_{l m} a_{i} a_{j}^{*} a_{l} a_{m}^{*}\right] d \mu_{\psi} \\
& =\int\left[\sum_{i \neq j}\left|A_{i j}\right|^{2}\left|a_{i}\right|^{2}\left|a_{j}\right|^{2}\right] d \mu_{\psi} \\
& =\frac{1}{e^{S}\left(e^{S}+1\right)} \sum_{i \neq j}\left|A_{i j}\right|^{2} \leq \frac{1}{e^{S}+1} \sigma_{\mathrm{ens}}^{2} .
\end{aligned}
$$

Here, in the second line, we used the fact that unless $i=m$ and $j=l$ the summand vanishes upon integration. In the third line, we used the fact that $\int d \mu_{\psi}\left|a_{i}\right|^{2}\left|a_{j}\right|^{2}=\frac{1}{e^{s}\left(e^{s}+1\right)}$ for $i \neq j$. A small subtlety in the final step is that

$$
\frac{1}{e^{S}} \sum_{i \neq j}\left|A_{i j}\right|^{2}=\operatorname{Tr}\left(\rho(P A P)^{2}\right)-\operatorname{Tr}(\rho A)^{2} \leq \sigma_{\mathrm{ens}}^{2} .
$$




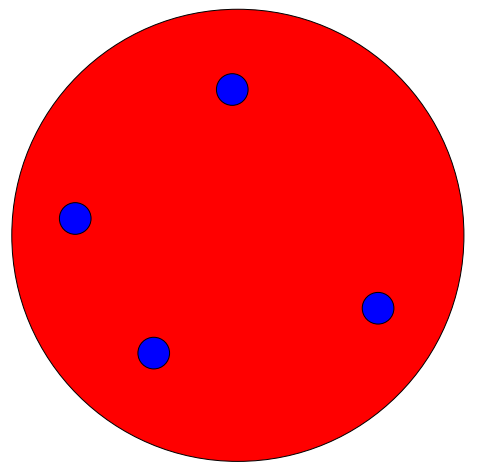

FIG. 2. The subspace, $H_{E}$, is a compact manifold. Most pure states in the space are very close to the maximally mixed state. An exponentially small volume of states (displayed in blue) can be a typical.

This difference arises because $A$ might have matrix elements that link states in $H_{E}$ to states outside $H_{E}$.

This result should be interpreted as follows. On almost all of the volume of the subspace, the expectation value of the operator differs from the typical expectation value by an exponentially small amount. The expectation value may differ significantly from the typical expectation value only in an expotentially small region of the subspace. The reader may consult Fig. 2 for intuition.

This result tells us that typical microstates of $H_{E}$ are described by a universal set of correlators. By itself, this does not disallow the possibility of an atypical basis of states for $H_{E}$. This is because the basis vectors themselves occupy only zero volume in the Hilbert space. We now bound the atypicality of a basis in some cases of interest.

Result 2: (Limit on atypicality of a basis) Assume that the ratio $\frac{\sigma_{\text {ens }}}{\langle A\rangle}$ vanishes as $\frac{1}{S^{\alpha}}$ for large $S$ and some positive number $\alpha$. Given any basis, $\left|f_{1}\right\rangle \ldots\left|f_{e^{s}}\right\rangle$, for $H_{E}$, let $\left|f_{\alpha_{1}}\right\rangle \ldots\left|f_{\alpha_{M}}\right\rangle$ be those of its elements where $\left|\frac{\left\langle f_{\alpha_{j}}|A| f_{\alpha_{j}}\right\rangle-\langle A\rangle}{\langle A\rangle}\right| \geq \mathrm{O}\left(\frac{1}{S^{\beta}}\right)$ in the thermodynamic limit, with $\beta<\alpha$. Then, $\frac{M}{e^{S}}$ vanishes at least as fast as $\mathrm{O}\left(\frac{1}{S^{2(\alpha-\beta)}}\right)$.

This result follows from simple inequalities,

$$
\begin{aligned}
\sigma_{\text {ens }}^{2}= & \frac{1}{e^{S}} \sum_{i}\left\langle f_{i}\left|A^{2}\right| f_{i}\right\rangle-\langle A\rangle^{2} \\
= & \frac{1}{e^{S}} \sum_{i}\left(\left\langle f_{i}\left|A^{2}\right| f_{i}\right\rangle-\left\langle f_{i}|A| f_{i}\right\rangle^{2}\right) \\
& +\frac{1}{e^{S}} \sum_{i}\left(\left\langle f_{i}|A| f_{i}\right\rangle-\langle A\rangle\right)^{2} \\
\geq & \frac{1}{e^{S}} \sum_{j=1}^{M}\left(\left\langle f_{\alpha_{j}}|A| f_{\alpha_{j}}\right\rangle-\langle A\rangle\right)^{2} \geq \frac{M \kappa^{2}\langle A\rangle^{2}}{e^{S}},
\end{aligned}
$$

where $\kappa=\inf _{j}\left|\frac{\left\langle f_{\alpha_{j}}|A| f_{\alpha_{j}}\right\rangle \mid-\langle A\rangle}{\langle A\rangle}\right|$. By assumption, $\kappa=\mathrm{O}\left(\frac{1}{S^{\beta}}\right)$ in the thermodynamic limit, and since $\frac{\sigma_{\text {ens }}^{2}}{\langle A\rangle^{2}}$ vanishes like $\frac{1}{S^{2 \alpha}}$, therefore $\frac{M}{e^{S}}$ must vanish like $\frac{1}{S^{2(\alpha-\beta)}}$.
The result above is very simple, but it is relevant for those observables that take on a finite classical expectation value. These are the observables for which $\frac{\sigma_{\text {ens }}}{\langle A\rangle}$ vanishes as $S \rightarrow \infty$. For such observables, the result rules out the existence of a basis whose individual elements give expectation values to the observable that are very different from the mean expectation value.

So far, our results have been kinematical. We now state a dynamical expectation about the spectrum, which should be true in almost all interacting systems. Let $S$ be the entropy at energy $E$. [We deliberately use the same notation as above since $e^{S}=\operatorname{dim}\left(H_{E}\right)$ if $H_{E}$ is taken to the subspace corresponding to the microcanonical ensemble.]

Expectation 1: (Almost continuous spectrum) The gap between the energy eigenvalues of typical neighboring high-energy eigenstates is $\mathrm{O}\left(e^{-S}\right)$ in an interacting theory in the thermodynamic limit.

The motivation for this expectation is as follows. In a finite band of energies $2 \Delta$, in the energy-range $[E-\Delta, E+\Delta]$, we expect to have $e^{S}$ states. Except for an exactly free theory, interactions generically break all degeneracies. Therefore, the energy gap between neighboring states scales like $e^{-S}$ in the thermodynamic limit.

Expectation 1 also holds in theories with supersymmetry. Supersymmetry might ensure that some states, which saturate the BPS bound, are degenerate. However, as soon as we move slightly away from the BPS bound, the gap between eigenvalues becomes exponentially small.

Some systems may have a forbidden zone of energies in which states cannot exist. For example, superconformal field theories may have Bogomol'nyi-Prasad-Sommerfield (BPS) representations that are separated from other representations with the same charges by a finite mass gap. (See, e.g., the b representations in Ref. [13].) However, outside the forbidden zone, we again expect exponentially small gaps between neighboring eigenvalues.

Expectation 1 also holds in integrable systems. The statistical mechanics literature contains considerable discussion of the statistics of the distribution of energy eigenstates. The statistics of eigenvalues differ in integrable and chaotic systems (see, e.g., Ref. [14].) But to our knowledge, except for free theories, the fact that the energy gap between typical eigenstates is $\mathrm{O}\left(e^{-S}\right)$ holds universally.

In the context of the fuzzball proposal, there has been some discussion that the correct gap between energy eigenstates, even at the supergravity point of the D1-D5 system, should be an inverse power of $N_{1} N_{5}$ (the product of the number of D1 and D5 branes) rather than an inverse exponential of this product $[15,16]$. This is based on the fact that at the orbifold point the D1-D5 conformal field theory (CFT) does have a gap that scales with $\frac{1}{N_{1} N_{5}}$. However, the orbifold CFT is a free theory. The moment we turn on the moduli that are necessary to reach the supergravity point, we expect that the degeneracies in the orbifold CFT will be destroyed. The entropy at energy 
$E$ scales as $S \propto \sqrt{N_{1} N_{5} E}$, and we expect that the gap between neighboring energy eigenstates is of order $e^{-S}$ at a generic point in moduli space.

The exponentially small gap can be easily detected by a two-point function. For example, let $A(t)$ be a simple operator localized in time. Then, given any typical highenergy basis state, $|f\rangle$ of energy $E$ (which may not be an eigenstate), consider

$$
G_{\widetilde{F}}\left(\omega_{0}\right)=\int d t\langle f|A(t) A(0)| f\rangle \mathfrak{F}_{\omega_{0}}(t) d t
$$

where $\mathfrak{F}_{\omega_{0}}(t)$ is a function of which the Fourier transform is centered around $\omega_{0}$ with a width $\delta \gg E e^{-S}$. But we can take $\delta$ to be very narrow. For example, in the D1-D5 theory, we may take $\delta=\frac{1}{\left(N_{1} N_{5}\right)^{4}}$ since this is still larger than $e^{-S}$. Then, by inserting a complete set of energy eigenstates, $\left|E_{i}\right\rangle$

$$
\begin{aligned}
G_{\widetilde{F}}\left(\omega_{0}\right)= & \sum_{i, j}\left\langle f \mid E_{i}\right\rangle\left\langle E_{i}|A(0)| E_{j}\right\rangle\left\langle E_{j}|A(0)| f\right\rangle \\
& \times\left[\int \mathfrak{\mho}_{\omega_{0}}(t) e^{i\left(E_{i}-E_{j}\right) t} d t\right] .
\end{aligned}
$$

Since the difference $E_{i}-E_{j}$ takes on almost a continuous range of values, we see that $G_{\widetilde{\gamma}}\left(\omega_{0}\right)$ has support for a continuous range of $\omega_{0}$. Even if the state $|f\rangle$ is a supersymmetric state, we can choose an appropriate operator $A$ that moves us off the BPS bound and of which the twopoint function displays a continuous spectrum.

States in which the two-point function does not have a continuous spectrum for any simple operator typically correspond to microstates of a phase of zero entropy. For such states, the three-point function $\left\langle E_{j}|A(0)| f\right\rangle$ that appears above vanishes for almost all except an exponentially small set of eigenstates, $\left|E_{j}\right\rangle$. For example, the boundary two-point function of light primary operators in the state dual to thermal AdS is expected to have a discrete spectrum.

In Ref. [17], it was argued that fuzzballs might represent typical states and still not show the continuous spectrum described above. Reference [17] suggested that the matrix elements $\left\langle E_{j}|A(0)| f\right\rangle$ could be subject to a selection rule: the matrix element vanishes unless $E_{j}-E=n E_{\text {gap }}$, where $E_{\text {gap }} \gg e^{-S}$ is some large gap and $n$ is an integer. Thus, probing a particular fuzzball microstate with simple operators only excites a tower of integrally spaced excitations on top of that microstate. A probe of another microstate excites a parallel tower, and it is impossible to move between towers by probing the system with simple operators. (See Fig. 3.) The number of towers must be exponentially large to account for the total number of states.

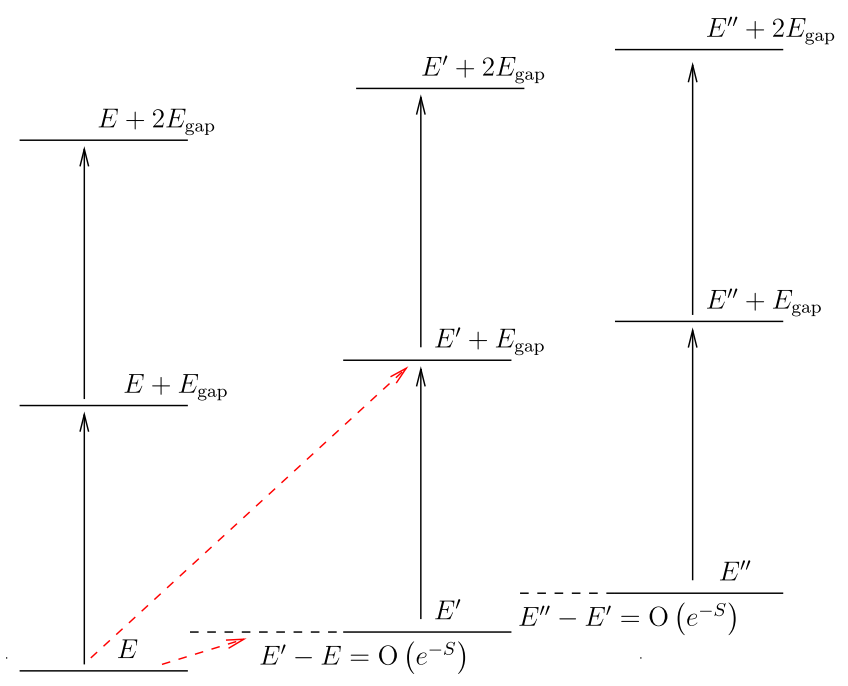

FIG. 3. An unusual possibility for the dynamics of fuzzball microstates. Probes of one microstate only excite other microstates in a single tower (solid black lines), and transitions between towers (dashed red lines) are disallowed.

This picture would suggest that the matrix elements between different states not only violate the eigenstate thermalization hypothesis (see below) but that most matrix elements actually vanish. Moreover, since it is impossible to transition between towers using simple operators, the system effectively breaks up into an exponentially large number of disconnected phases. If the D1-D5 system or any gravitational system shows such unusual statistical behavior, there should be a dynamical explanation for this. The selection rule above cannot just be postulated to save the fuzzball program from potential contradictions. In the absence of such a dynamical explanation, the simplest possibility is just that fuzzballs and other states in which the two-point function does not show a continuous spectrum represent isolated states of which the degeneracy is exponentially small compared to the set of all microstates.

\section{Relation to eigenstate thermalization}

Before we conclude our discussion on general statistical expectations, we should clarify the relation to the commonly discussed eigenstate thermalization hypothesis (ETH) [18].

The ETH is usually stated for energy eigenstates. However, it really only relies on the assumption that in a large statistical system the eigenstates of most observables are likely to be highly scrambled versions of eigenstates of the Hamiltonian. So, given some basis of states for the microcanonical ensemble, $\left|f_{i}\right\rangle$, the ETH can be stated as

$$
\left\langle f_{j}|A| f_{i}\right\rangle=A\left(E_{i}\right) \delta_{i j}+B\left(\frac{E_{i}+E_{j}}{2}\right) e^{\frac{-S}{2}} R_{i j},
$$


where $\mathrm{R}$ is a matrix of random phases and $E_{i}$ and $E_{j}$ are the expectation values of the Hamiltonian in $\left|f_{i}\right\rangle$ and $\left|f_{j}\right\rangle$ and $A$ and $B$ are smooth functions of their arguments. Note that $A(E)$ is the microcanonical average of the observable at energy $E$.

If the ETH holds for some basis of states, this implies that most elements of such a basis are typical even for operators in which the standard deviation is not parametrically small in the thermodynamic limit.

The ETH is a significantly stronger assumption than the vanishing of the microcanonical standard deviation for classical observables that is an input to Result 2 The ETH implies the typicality of most elements of the basis even for observables that have large $\sigma_{\text {ens }}$. The ETH arises from an assumption of quantum chaos, and in such a system, expectation 1 also holds.

Therefore, the ETH is stronger than the assumptions of Result 2 and Expectation 1. Nevertheless, we expect the ETH to hold in theories with holographic duals [19].

\section{B. Implications for the fuzzball program}

Now, we discuss several implications of these results for the fuzzball program. In what follows, to make contact with the results above, we take the Hilbert space $H_{E}$ to be the subspace that corresponds to black holes. If we consider large black holes in the AdS/CFT correspondence, then this subspace can simply be taken to be the microcanonical ensemble. However, note that the subspace corresponding to black holes exists even in flat space, where black holes do not dominate the canonical or microcanonical ensemble.

\section{Information-free nature of the horizon/stretched horizon}

The fuzzball program is often motivated by the idea that the horizon should be replaced by the surface of a fuzzball that would contain "information" about the initial state. It is claimed that this structure would correct Hawking radiation at $\mathrm{O}(1)$.

We now apply Result 1 . Let $A$ correspond to an operator that measures correlations between different Hawking quanta. For example, $A$ may be a product of curvature invariants at distinct points. Since we are considering smooth geometries, such invariants are bounded, and their fluctuations cannot be exponentially large. Then, Result 1 tells us that such observations in a typical microstate yield only an exponentially small amount of information.

In particularly, it cannot be the case that Hawking radiation differs by $\mathrm{O}(1)$ amounts between different typical microstates. It is sometimes claimed that "high-energy" observables would take on a universal form but "lowenergy" observables at the scale of the Hawking radiation would differ between microstates [20]. However, Result 1 allows no such freedom. In a typical microstate, both highenergy and low-energy observables take on a universal value, and all features of the microstate can only be determined by exponentially precise observations.

The idea that the surface of a black hole should contain "information" is often presented by making an analogy with a piece of coal. (For instance, see page 3 of Ref. [6].) When coal burns, the properties of the outgoing radiation are strongly affected by the nature of its surface. However, this is a misleading analogy; everyday pieces of coal are not completely thermalized. They have a number of distinctive features because they are in highly atypical states. A better example, to visualize a thermalized system, is a gas of radiation in a box. This gas is entirely featureless. Individual photons that emerge from the box contain almost no information about the state of the radiation inside the box; it is only by making exponentially precise measurements on the radiation that we can discern the state of the radiation.

\section{Universal fuzzball geometry}

Above, we argued that correlation functions of Hawking radiation measured in a typical microstate must take on a universal value. We now argue that these correlators should correspond to correlators computed in effective field theory about an approximately classical average bulk geometry in the limit in which the Planck length is smaller than all other scales.

The signature of an approximately classical bulk geometry is that correlators of local operators factorize into products of lower-point functions [21].

In AdS/CFT, the factorization of boundary correlators in the microcanonical/canonical ensemble can be proved at large $N$ using the standard factorization arguments. We can then use the standard Hamilton-Kabat-Lifschytz-Lowe construction [22] to construct approximately local operators, and the factorization of boundary correlators implies the factorization of bulk correlators. This implies that, in AdS/CFT, the microcanonical/canonical ensemble is dual to an approximately classical bulk geometry. By Result 1 , this is also the geometry dual to a typical microstate. This geometry can also be used to compute $n$-point functions of simple operators to excellent accuracy.

In flat space, we cannot make such a clear argument that averages computed in $H_{E}$ correspond to an approximately classical bulk. But, even here, we expect that S-matrix elements will factorize if the Planck length is much smaller than other length scales in the problem. These S-matrix elements can be used to reconstruct a bulk geometry that is approximately classical.

A priori, we do not know what this universal classical geometry should be. In Sec. III, we will compute this average geometry for the two-charge Lunin-Mathur solutions that have been quantized. However, in more general settings that correspond to large black holes, we cannot compute this average geometry since all fuzzball solutions have neither been found nor quantized. So, in the remainder 
of this paper, we will simply proceed with the following expectation.

Expectation 2: (Conventional geometry as average) The conventional black-hole geometry-after incorporating classical string-theory corrections-correctly computes the average value of bulk observables such as the metric and correlation functions of the metric as long as we are more than the Planck length outside the horizon.

We believe that this is a fairly uncontroversial assumption. If the geometry obtained by averaging over all microstates differs significantly from the black-hole geometry, this has significant implications for AdS/CFT: it would imply the computations in a thermal state in the CFT should be matched to bulk computations in this special average fuzzball geometry (whatever it may be) rather than the black hole. This would be the case even for timeordered correlators that are obtained naturally from Euclidean computations. Therefore, any claim that Expectation 2 is violated must be accompanied by an explanation for why the Euclidean saddle point is not adequate for correlators outside the horizon. We are not aware of any place in the literature in which such a strong claim has been made.

Expectation 2 allows for the possibility that the average geometry has Planck-scale deviations from the conventional geometry. We discuss these deviations in greater detail below.

\section{Distinct fuzzballs as a basis?}

Result 1 implies that the geometries corresponding to typical states can only differ by an exponentially small amount from the average geometry. Since these differences are much smaller than the quantum fluctuations about the geometry, we do not expect to be able to represent such exponentially small deviations in terms of distinct classical metrics. So, the idea that typical microstates are represented by geometrically distinct fuzzballs seems untenable to us. Typical microstates are represented by the same average geometry. ${ }^{1}$

One might imagine that, while it is impossible to describe the different typical microstates using geometries, perhaps one could use a set of distinguishable geometries as a basis for all microstates of the black hole. However, we will now show that Result 2, together with Expectation 2, constrains how much the typical element of the basis can differ from the conventional black hole. To make this

\footnotetext{
${ }^{1}$ We note that in the examples, in which a precise description of microstates is available, their differences lie in properties of the quantum wave functions of a dual set of nongravitational variables. If a gravitational description of microstates is possible, we would expect that their differences lie in the nonperturbative properties of the quantum wave function that describes fluctuations of the metric and all other degrees of freedom in the theory and not in differences at the level of the geometry.
}

precise, we pause to define some useful intermediate quantities that we will use later in the paper as well.

\section{4. "Difference" and "quantumness" parameters}

Let $\hat{O}(r)$ be a simple bulk observable. For example, $\hat{O}(r)$ may be some coordinate invariant function of the metric. Here, $r$ denotes the "radial" coordinate in a coordinate system in which the horizon is at $r=r_{h}$ and $r=\infty$ is the asymptotic region. To make physical meaningful comparisons, $r$ should be defined through the physical area of a compact submanifold in the geometry.

Let $O^{\mathrm{bh}}(r)$ be the expectation value of this observable in the black hole. For a fuzzball microstate, $|f\rangle$, we denote

$$
\langle f|\hat{O}(r)| f\rangle=O^{\mathrm{fuzz}}(r) .
$$

The quantum fluctuations of this operator, in the fuzzball state, are measured by

$$
\sigma^{2}(r)=\left\langle f\left|\hat{O}(r)^{2}\right| f\right\rangle-\langle f|\hat{O}(r)| f\rangle^{2},
$$

where the product of operators at a point may need to be suitably renormalized.

We now define two parameters. The difference parameter, $\mathrm{d}$, is defined as

$$
\mathrm{d}_{O}(r)=\left|\frac{O^{\mathrm{bh}}(r)-O^{\mathrm{fuzz}}(r)}{O^{\mathrm{fuzz}}(r)}\right| .
$$

The quantumness parameter, $\mathbf{q}$, is defined as

$$
\mathrm{q}_{o}(r)=\left|\frac{\sigma(r)}{O^{\text {fuzz }}(r)}\right| .
$$

For a classical solution to be "interesting," we require that the difference parameter is large. On the other hand, for the classical solution to be reliable, the quantumness parameter must be parametrically suppressed. This is particularly important in a nonlinear theory like gravity. It makes no sense to trust classical general relativity in a regime in which quantum fluctuations of the metric are of the same order as the metric itself.

We argue below that typical fuzzballs cannot meet both conditions simultaneously. In the region where they are interesting, they also become unreliable.

\section{Deviations of individual fuzzballs from the average geometry}

From Result 1, the fluctuations that enter Result 2 are the same as quantum fluctuations in a typical state. Since we argued above that typical states correspond to the conventional black-hole geometry, we can estimate the fluctuations that enter Result 2 by estimating quantum fluctuations in the black-hole geometry, 


$$
\begin{aligned}
\sigma_{\text {ens }}(r) & =\frac{1}{e^{S}} \operatorname{Tr}_{H_{E}} \hat{O}(r)^{2}-\left(\frac{1}{e^{S}} \operatorname{Tr}_{H_{E}} \hat{O}(r)\right)^{2} \\
& =\left\langle\Psi\left|\hat{O}(r)^{2}\right| \Psi\right\rangle-\langle\Psi|\hat{O}(r)| \Psi\rangle^{2}+\mathrm{O}\left(e^{-\frac{S}{2}}\right),
\end{aligned}
$$

where $|\Psi\rangle$ is a typical microstate, and we have used Result 1 in the second equality. Note that $\sigma_{\text {ens }}(r)$ might not coincide with $\sigma(r)$ defined above if $|f\rangle$ is not a typical state.

The leading quantum fluctuations in the black-hole geometry appear with a factor of $\frac{1}{G_{N}}$, and on dimensional grounds, we expect that they are proportional to $\left(\frac{\ell}{\ell_{\mathrm{pl}}}\right)^{d-2}$, where $\ell$ is the typical length scale in the geometry. If we are far away from the horizon, then we expect that $\ell \leq r_{h}$. We also note that the entropy is proportional to $\left(\frac{r_{h}}{\ell_{\mathrm{pl}}}\right)^{d-2}$. Therefore, for simple gauge-invariant observables made out of the metric, we expect that for observables with a nonzero classical expectation value ${ }^{2}$

$$
\frac{\sigma_{\mathrm{ens}}^{2}(r)}{\left(O^{\mathrm{bh}}(r)\right)^{2}}=\mathrm{O}\left(\frac{1}{S}\right), \quad r-r_{h} \gg \ell_{\mathrm{pl}}
$$

(The reader may consult Ref. [23] for a concrete calculation of quantum fluctuations in the black-hole background.)

However, then, Result 2 tells us that for all but a vanishing fraction of fuzzball states we also have

$$
\mathrm{d}_{O}(r)=\left|\frac{O^{\mathrm{bh}}(r)-O^{\mathrm{fuzz}}(r)}{O^{\mathrm{fuzz}}(r)}\right|=\mathrm{O}\left(\frac{1}{\sqrt{S}}\right), \quad r-r_{h} \gg \ell_{\mathrm{pl}} .
$$

Moreover, if $\mathrm{d}_{O}$ is very small, then quantum fluctuations of the metric in the fuzzball geometry are also very close to quantum fluctuations in the black-hole geometry. Therefore,

$$
\mathrm{q}_{O}(r)=\mathrm{O}\left(\frac{1}{\sqrt{S}}\right)
$$

So, the deviation of the fuzzball metric from the black-hole metric can at most be of the same order as the quantum fluctuations of the metric.

It is important that (2.8) continues to hold when $r=r_{h}+\ell_{\text {str }}$, where $\ell_{\text {str }}$ is the string length. The blackhole metric is corrected at the string scale, but we can compute fluctuations of the metric, using Euclidean quantum gravity, and we do not expect quantum fluctuations in

\footnotetext{
${ }^{2}$ What we will need, in subsequent sections, is just that $\frac{\sigma_{\text {ens }}(r)}{O^{\mathrm{b} h}(r)}$ is small—not that it takes the precise value predicted by the blackhole geometry. This may hold even if Expectation 2 fails; as long as the typical microstate in $H_{E}$ corresponds to an approximately classical geometry, we can estimate $\sigma_{\text {ens }}$ by quantizing metric fluctuations in this geometry, and these quantum fluctuations will be small provided that typical curvatures are small.
}

the black-hole geometry to become significant at the string scale.

Since, by definition, fuzzballs have no horizon, they must start to deviate appreciably from the conventional black-hole geometry at some point. The argument above tells us that for typical fuzzballs this can only happen when $r-r_{h}=\mathrm{O}\left(\ell_{\mathrm{pl}}\right)$. This is precisely when Expectation 2 also allows the average geometry to deviate from the conventional geometry. ${ }^{3}$

But this means that the geometric solution-corresponding to a typical basis state or the average geometry-must explicitly have Planck-scale structures, presumably through an explicit length scale that takes on a Planck-scale value. However, we expect that any length scale in quantum gravity will itself undergo fluctuations of the size of the Planck scale. Therefore, in the region where we are very close to the horizon, if the fuzzball has explicit Planck-scale features, then quantum fluctuations in the metric are expected to be of the same order as these Planck-scale structures. So,

$\mathrm{d}_{O}(r)=\mathrm{O}(1), \quad$ but $\mathrm{q}_{O}(r)=\mathrm{O}(1)$, when $r-r_{h}=\mathrm{O}\left(\ell_{\mathrm{pl}}\right)$.

But if the parameter $\mathrm{q}_{o}=\mathrm{O}(1)$, then the classical solution becomes completely unreliable. So, if we explicitly insert Planck-scale features into the fuzzball solution in order to satisfy Result 2, then we run into the difficulty that the geometry becomes unreliable just where it appears to be interesting.

To summarize, we have argued the following. If fuzzballs are to represent typical microstates, then they must have the following features:

(1) When we are far away from the horizon (in Planck units), the fuzzball geometry is indistinguishable from the black-hole geometry up to terms that are suppressed by the black-hole entropy. This follows from the fact that the average fluctuations of the metric-which can be computed in the black-hole geometry-are small, and then Result 2 limits the extent to which typical basis elements can differ from the average.

(2) When we approach within a Planck length of the horizon, the fuzzball geometry may appear to deviate from the conventional black hole. But

\footnotetext{
${ }^{3}$ Standard calculations in the conventional black-hole geometry suggest that when the geometry has a macroscopic horizon we do not expect any unusual effects in the near-horizon region and $\sigma_{\text {ens }}$ continues to be small there. However, it is difficult to prove this even in holography, since the Hamilton-KabatLifschytz-Lowe construction requires very long time bands on the boundary to represent physics in the region $r-r_{h}=\mathrm{O}\left(\ell_{\mathrm{pl}}\right)$. The length of these bands scales with $N$ and may interfere with standard large- $N$ counting. So, in this paper, we make a generous assumption for the fuzzball program by allowing the possibility that some hitherto unknown effect invalidates the standard calculation of $\sigma_{\text {ens }}$ within a Planck length of the horizon and somehow makes it large.
} 


$$
r=r_{h}+\ell_{\mathrm{pl}}
$$

FIG. 4. A schematic representation of what a typical fuzzball geometry must look like, if fuzzballs represent black-hole microstates. The geometry must closely resemble the black-hole geometry away from the horizon (unshaded region) and then suddenly deviate away to cause some extra dimension to pinch off when we reach within the Planck length of the horizon (blue region).

such a geometry must explicitly contain Planckian structures, and then we expect that quantum fluctuations will become large and so the fuzzball geometry will become unreliable. (Note that this is contrast to the conventional black-hole geometry, which remains perfectly reliable close to the horizon.)

This picture of the fuzzball is shown schematically in Fig. 4.

Therefore, it is wrong to think of fuzzballs as macroscopically distinct geometries, which somehow average out to give the same answer as the black hole. Rather, typical fuzzballs must all look like Fig. 4 to satisfy Result 2 . Fuzzballs which have structure on a scale larger than the Planck scale can only be a vanishing fraction of microstates by Result 2 .

\section{Requirement of large redshifts}

The discussion above utilizes Expectation 2, which is what leads to Eq. (2.7). However, even without invoking Expectation 2, we can still use Expectation 2 to justify the important aspects of the picture shown in Fig. 4.

Consider a quantum field that propagates in the bulk of which the excitations about the vacuum are gapped. In global AdS, we can consider a massless field. If we are considering flat space or Poincaré AdS, we can consider a massive field. Then, Expectation 1 implies that the asymptotic two-point function of this field must be supported at arbitrary frequencies even though, locally, field excitations are gapped.

The reason that black holes allow this phenomenon is because of the infinite redshift at the horizon. This redshift allows for arbitrary low-energy excitations. This is not a bug; it is a feature of the black-hole geometry which ensures that it can be interpreted as a heavy state in a quantum-mechanical system with large entropy.

If fuzzballs are to represent black-hole microstates, they must also support a continuous spectrum. Therefore, the fuzzball geometry must also have an extremely large shift. In particular, if the geometry caps off to form a fuzzball at any length scale that is visible classically, then the inverse of this length scale will be visible as an energy gap that would violate Expectation 1. Once again, we see that the requirement of an almost continuous spectrum disallows fuzzballs that are of size $\ell_{\text {str }}$ or any other classical length scale.
What if the geometry caps off at $r=r_{h}+\ell_{\mathrm{pl}}$ ? Even such a geometry would not support the exponentially suppressed gap that is required around a heavy pure state since it would allow, at most, an energy gap that is power-law suppressed in the entropy. So, the only possibility is for the geometry to stop making sense classically below $r=r_{h}+\ell_{\mathrm{pl}}$. However, if this happens, we return to our conclusion above, displayed in Fig. 4: fuzzballs are uninteresting in most of space (where $r-r_{h} \gg \ell_{\mathrm{pl}}$ ) and unreliable where they are interesting $\left[r-r_{h}=\mathrm{O}\left(\ell_{\mathrm{pl}}\right)\right]$.

Larger fuzzballs that are both reliable and interesting by virtue of having larger than Planck-scale structure are irrelevant to the discussion of black-hole microstates since they do not have the right energy gap expected in a system with large entropy.

\section{Eigenstate thermalization}

The arguments that led to the structure expected from typical fuzzball geometries shown in Fig. 4 assumed that the bulk metric was a good observable with small quantum fluctuations. We believe that this is a very robust assumption.

However, if we assume the ETH, then we can deduce such a structure for typical fuzzballs while restricting our discussion to only asymptotic observables. If we apply the ETH in the form (2.4) to fuzzball states, then we expect to get the microcanonical average

$$
\langle f|A| f\rangle=A(E)
$$

where $E$ is the energy of the fuzzball state and $A(E)$ is the microcanonical average of $A$.

Therefore, the ETH tells us that, even for asymptotic observables, we should get precisely the same value in a typical fuzzball microstate as we do in the conventional black hole.

Intuitively, this rules out fuzzballs that differ at leading order from the black-hole metric. This is because if the geometry differs at leading order, then a simple scattering experiment with waves sent in from asymptotic infinity will detect this variation and produce an answer that fails to satisfy the ETH. We will see in Sec. IV that this is precisely what happens for fuzzballs with macroscopic structures. 


\section{Cautionary note}

We close this subsection with a note of caution. The reader will note that our arguments above have been based on simple physical expectations and general results from statistical mechanics. This makes them broadly applicable, but it also means that these arguments are only suggestive of the difficulties that the fuzzball program must surmount and cannot be taken as a proof that the program is not viable.

In Secs. III and IV, we will verify the correctness of these arguments in specific examples. But if the fuzzball program is to be carried through to completion in any system, this must involve a loophole in the arguments outlined above, and it would be interesting to understand the origins of such a loophole.

\section{Indirect arguments for horizon structure}

In an attempt to sidestep arguments of the kind that we have provided above, Mathur put forward indirect arguments to show that the horizon must have structure [7]. Mathur's argument was based on the strong subadditivity, and it was later used to produce the firewall paradox [24].

This argument has been reviewed several times, and, in particular, we refer the reader to Sec. 6.1 of Ref. [25] for an up-to-date review. Very briefly, the idea is to divide a nice slice in the late part of an evaporating black hole into a near horizon region outside the horizon, $B$; the rest of the region outside the black hole, $A$; and a near-horizon region inside the horizon, $C$.

An argument due to Page [26] suggests that in the latter part of black-hole evaporation $S_{A B}<S_{A}$, whereas the smoothness of the interior suggests that $S_{B C}<S_{C}$. These two inequalities are in contradiction with the strong subadditivity of entropy $S_{A B}+S_{B C} \geq S_{A}+S_{C}$.

In Ref. [7], this was used to argue that we should drop $S_{B C}<S_{C}$ and consequently that we should drop the idea that the interior of the black hole is a smooth featureless region.

The difficulty with this argument is that the strong subadditivity of entropy assumes that the set of observables on a nice slice factorizes into observables on $A, B$, and $C$. However, in quantum gravity, this is simply not true. On the contrary, observables in $C$ can be written as scrambled versions of observables in $A$.

We emphasize that this is not some hand-waving argument about complementarity. This phenomenon-in which local operators in one region can be rewritten as complicated local operators in another region-can be made absolutely precise in empty AdS [27]. Moreover, it was shown in Ref. [25] that if we take strong subadditivity seriously in quantum gravity we can construct paradoxes even in empty AdS (see Sec. 6.4 of Ref. [25].)

Even in flat space, where the explicit formulas of Ref. [27] cannot be written down, we have substantial evidence from the dynamical breakdown of string perturbation theory in some regimes that a similar loss of locality occurs at a nonperturbative level in quantum gravity $[25,28]$.

The arguments of Ref. [7] were preceded by other arguments that the information paradox requires a smooth horizon to be replaced by a geometry with details that would carry information about the initial state. These arguments are unsatisfactory because it is well understood that information can be stored in exponentially small correlations between different Hawking quanta [29]. So, the recovery of information outside the black hole does not, by itself, require any large modifications to the classical black-hole geometry.

A related set of ideas suggests that a pure state cannot have a horizon because a horizon has entropy, whereas pure states cannot have entropy [30]. However, this is incorrect. The thermodynamic entropy should not be conflated with the von Neumann entropy. So, pure states can also have thermodynamic entropy, which arises after we coarse grain the system, and this entropy reflects the fact that coarsegrained probes of the system leave its fine-grained features undetermined [31]. In a theory of quantum gravity, the geometry is a tool to encode expectation values of the metric and its low-point correlators-it is thus an explicitly coarse-grained probe of the full theory. So, it is perfectly consistent for the geometry to be described by a metric with a horizon of which the entropy reflects the fact that we have ignored the fine-grained nonmetric degrees of freedom that are part of the full description.

We note, parenthetically, that in anti-de Sitter space Ref. [32] made an entirely independent set of arguments to suggest that the black-hole interior cannot be represented in the boundary CFT. These arguments are relevant for large AdS black holes that are thermodynamically stable. Moreover, even if they are correct, they suggest that the black hole has a horizon and the interior of a black hole has a firewall rather than a fuzzball. For this reason, the arguments of Ref. [32] are not directly relevant here, and a detailed discussion of their merits is beyond the scope of this paper. Nevertheless, a brief summary of their status is as follows.

Several authors [33-35] have pointed out that interior operators can be constructed using a suitably state-dependent construction. The authors of Ref. [36] suggested that state dependence would lead to observable peculiarities for an infalling observer, but it was explained in Ref. [37] that these effects were not observable in physically reasonable experiments.

Physically, our understanding of the origins of state dependence has also advanced. The state dependence of the interior can be understood as arising from a fat tail in the inner product of coherent states in gravity [34], and this fat tail also contributes to the fact that interior operators, when gauge fixed in a particular manner, may fail to satisfy a nonperturbative version of the Hamiltonian constraints 
[38]. The origins of state dependence can also be studied in toy models [39]. Of course, several questions about state dependence and the reconstruction of the black-hole interior in AdS/CFT remain to be understood.

To summarize this subsection, we have argued that indirect arguments for the relevance of fuzzballs to black holes are invalid. This is an important point. It shows that one cannot concede the limitations of supergravity solutions-as is sometimes done in the fuzzball program - but yet argue that black holes do not have smooth interiors. Neither a resolution to the information paradox nor an understanding of the black-hole entropy requires the existence of fuzzballs. The relevance, or lack thereof, of fuzzballs to the study of black holes must follow from a study of the known fuzzball solutions. If these solutions are irrelevant to black holes, there is no other valid argument for the relevance of fuzzballs for black holes.

\section{QUANTUM ASPECTS OF THE TWO-CHARGE SOLUTIONS}

In this section, we examine the original two-charge fuzzball solutions that were discovered in Refs. [4,5]. The literature on these solutions and their relation to CFT microstates is extensive. Our analysis will be simple and independent, but we note a few salient results in the literature. In Ref. [40] and then in Ref. [41], it was pointed out that the supergravity solution was not valid for typical states and as one travels toward the fuzzball cap it is necessary to transition out of the D1-D5 duality frame. This can indeed be done in some cases, and for specific solutions, the full stringy description was analyzed in Ref. [42]. We will reach a similar conclusion, although our reasoning will be slightly different and placed within the framework developed in Sec. II.

There has also been work on identifying specific solutions with microstates in the orbifold CFT [43]. We note that, even for very simple states, such an identification must be performed carefully since the supergravity point is very far from the orbifold point in the D1-D5 moduli space and, moreover, states cannot be uniquely identified just by specifying one-point functions of a few operators. In fact, in general, such an identification is impossible since the matching between states at different points in moduli space is path dependent [44]. Holographic correlators have also been calculated in these solutions [45] as we will do for multicharge solutions in Sec. IV.

For us, what is important is that these solutions were quantized in Ref. [3], following a suggestion made in Ref. [46]. Therefore, we can study the quantum mechanics of this set of solutions, and we will use this system to verify the arguments of Sec. II. We will compute the average fuzzball geometry, and we will also compute quantum fluctuations in this geometry. This allows us to compute the parameters $d$ [defined in (2.5)] and q [defined in (2.6)]. This system differs slightly from the setup of Sec. II because the horizon of the conventional solution is of zero size because a circle in the geometry shrinks to zero at that point. Nevertheless-using the size of this circle as a measure of the distance from this zero-size horizon-we find the following:

(1) As the distance from the position of the conventional horizon becomes greater than the Planck length, the average fuzzball geometry tends rapidly to the classical geometry.

(2) The average geometry starts deviating from the conventional geometry when we are within the Planck length, and not the string length, of the horizon. Moreover, most of the entropy of the set of solutions comes from solutions that differ from the conventional geometry at the Planck scale.

(3) In the region where deviations of the average geometry from the conventional geometry are appreciable, quantum fluctuations are of the same order as the expectation values of components of the metric. Therefore, the solution is entirely unreliable.

We will consider the two-charge solutions in the following form, using the conventions of Ref. [3]:

$$
\begin{aligned}
d s^{2} & =e^{-\frac{\phi}{2}} d s_{\mathrm{str}}^{2} ; \quad e^{-2 \phi}=\frac{f_{5}}{f_{1}} ; \\
d s_{\mathrm{str}}^{2} & =\frac{1}{\sqrt{f_{1} f_{5}}}\left(-(d t+A)^{2}+(d y+B)^{2}\right)+\sqrt{f_{1} f_{5}} d \vec{x}^{2}+\sqrt{\frac{f_{1}}{f_{5}} d \vec{z}^{2}} \\
f_{5} & =1+\frac{Q_{5}}{L} \int_{0}^{L} \frac{d s}{|\vec{x}-\vec{F}(s)|^{2}} ; \quad f_{1}=1+\frac{Q_{5}}{L} \int_{0}^{L} \frac{\left|\vec{F}^{\prime}(s)\right|^{2}}{|\vec{x}-\vec{F}(s)|^{2}} ; \\
A_{i} & =\frac{Q_{5}}{L} d x^{i} \int_{0}^{L} \frac{F_{i}^{\prime}(s)}{|\vec{x}-\vec{F}(s)|^{2}} d s ; \quad d B=*_{4} d A ; \\
C & =\frac{1}{f_{1}}(d t+A) \wedge(d y+B)+\mathcal{C} ; \quad d \mathcal{C}=-*_{4} d f_{5} .
\end{aligned}
$$


Here, $\vec{z}$ denotes four compact directions. The conventional solution is obtained simply by setting

$$
f_{1} \rightarrow 1+\frac{Q_{1}}{\vec{x}^{2}} ; \quad f_{5} \rightarrow 1+\frac{Q_{5}}{\vec{x}^{2}}
$$

and setting $A=0, B=0$.

These solutions can be systematically quantized by recognizing that the space of classical solutions can be bijectively mapped to points on the phase space; the action of the theory yields a symplectic form on this space, and the machinery of geometric quantization can then be applied to obtain a Hilbert space [47]. The result of this process is very simple. The quantization promotes the functions $F^{k}(s)$ to operators as

$$
F^{k}(s)=\mu \sum_{n>0} \frac{1}{\sqrt{2 n}}\left(a_{n}^{k} e^{\frac{-2 \pi \text { ins }}{L}}+\left(a_{n}^{k}\right)^{\dagger} e^{\frac{2 \pi \text { ins }}{L}}\right),
$$

where $\left[a_{n}, a_{m}^{\dagger}\right]=\delta_{n m}$. The various parameters that appear here are defined as

$$
\mu=\frac{g_{s}}{R \sqrt{V_{4}}} ; \quad L=\frac{2 \pi Q_{5}}{R} .
$$

Here, $R$ is the coordinate radius of the $y$ direction, and $V_{4}$ is the coordinate volume of the compact manifold. These are moduli of the solution. We are working in units in which the string length is set to unity. The charges are related to the brane numbers by

$$
Q_{5}=g_{s} N_{5} ; \quad Q_{1}=\frac{g_{s} N_{1}}{V_{4}} .
$$

For the purposes of counting states, it will be useful to define the "Hamiltonian"

$$
H=\sum_{n>0, k} n\left(a_{n}^{k}\right)^{\dagger} a_{n}^{k},
$$

where we have a infinite set of harmonic oscillators with creation and annihilation operators specified by $a_{n}^{k}$ and $k$ runs over 1...4. The fuzzball states dual to the D1-D5 system with charges $\left(Q_{1}\right.$ and $\left.Q_{5}\right)$ are defined to be the states in this quantum system that have $H=N_{1} N_{5}$.

We will not attempt to compute the full quantum expectation value of the metric. Instead, we will focus on the list of quantum expectation values

$$
\left\langle\Psi\left|f_{5}-1\right| \Psi\right\rangle, \quad\left\langle\Psi\left|f_{1}-1\right| \Psi\right\rangle, \quad\left\langle\Psi\left|A_{i}\right| \Psi\right\rangle,
$$

in a typical state, $|\Psi\rangle$. Here, "typical state" is used in the sense of Result 1 . These one-point expectation values were also calculated in Ref. [48], and our results agree precisely with those. We will not consider $B_{i}$ separately since this field is defined through the dual of $A$. Note that we also subtract off the uninteresting 1 in both $f_{1}$ and $f_{5}$.

$A_{i}$ vanishes in the conventional geometry, and it will turn out that $\left\langle\Psi\left|A_{i}\right| \Psi\right\rangle$ also vanishes. So, the difference and quantumness parameters [Eqs. (2.5) and (2.6)] are not well defined for this observable. Therefore, we will consider another 1-form that does not appear in the metric but is also an interesting probe of the geometry

$$
W_{i}=\frac{Q_{5}}{L} \int_{0}^{L} \frac{F_{i}(s)}{|\vec{x}-\vec{F}(s)|^{2}} d s .
$$

This quantity is of interest since it vanishes in the conventional geometry, but, as we will find, $\left\langle\Psi\left|W_{i}\right| \Psi\right\rangle$ takes on a finite value. So, one can ask if this finite value is reliable.

We will also compute the quantum fluctuations in these quantities by computing the following quantum two-point functions:

$\left\langle\Psi\left|\left(f_{5}-1\right)^{2}\right| \Psi\right\rangle, \quad\left\langle\Psi\left|\left(f_{1}-1\right)^{2}\right| \Psi\right\rangle, \quad\left\langle\Psi\left|W_{i} W_{j}\right| \Psi\right\rangle$.

We will use these two-point functions to evaluate the difference and quantumness parameters for these observables. These calculations will allow us to verify all the expectations outlined in Sec. II in a precise setting.

\section{A. One-point functions:}

$\left\langle\Psi\left|f_{5}-\mathbf{1}\right| \Psi\right\rangle,\left\langle\Psi\left|f_{1}-\mathbf{1}\right| \Psi\right\rangle,\left\langle\Psi\left|A_{i}\right| \Psi\right\rangle,\left\langle\Psi\left|W_{i}\right| \Psi\right\rangle$

Using Result 1, the expectation value of the observables above in a typical state can be computed by considering the microcanonical trace. Therefore, we can consider the generating function

$\mathcal{G}(\chi, \alpha)=\frac{1}{e^{S(E)}} \operatorname{Tr}_{\text {mic }}\left[\int_{-\infty}^{\infty} \prod_{k} d g_{k} \int_{0}^{\infty} d t \int_{0}^{L} \frac{d s}{L}: e^{\mathcal{F}}:\left(\frac{t}{\pi}\right)^{2}\right]$,

where

$$
\begin{aligned}
\mathcal{F}= & -\sum_{k} t_{k} g_{k}+2 i t\left(x^{k}-F^{k}(s)\right) g_{k} \\
& +\sum_{j} \alpha^{j} F^{j}(s)+\chi^{j} \frac{d F^{j}(s)}{d s} .
\end{aligned}
$$

Here, the trace is taken over all energy eigenstates of the Hamiltonian with a large total energy,

$$
E=N_{1} N_{5},
$$

and the degeneracy of states at that energy is given by $e^{S(E)}$. By normal ordering, we mean that when we expand the exponential in terms of creation and annihilation operators we move all annihilation operators to the right. This, of course, involves a necessary choice of how to interpret the quantum operator corresponding to the classical quantity. 
From Result 1, in a typical microstate $|\Psi\rangle$, we expect

$$
\begin{aligned}
\left\langle\Psi\left|f_{5}\right| \Psi\right\rangle & =1+Q_{5} \mathcal{G}(\chi=0, \alpha=0) ; \\
\left\langle\Psi\left|f_{1}\right| \Psi\right\rangle & =1+Q_{5} \lim _{\chi \rightarrow 0} \frac{\partial}{\partial \chi^{i}} \frac{\partial}{\partial \chi^{i}} \mathcal{G}(\chi, \alpha=0) ; \\
\left\langle\Psi\left|A_{i}\right| \Psi\right\rangle & =Q_{5} \lim _{\chi \rightarrow 0} \frac{\partial}{\partial \chi^{i}} \mathcal{G}(\chi, \alpha=0) ; \\
\left\langle\Psi\left|W_{i}\right| \Psi\right\rangle & =Q_{5} \lim _{\alpha \rightarrow 0} \frac{\partial}{\partial \alpha^{i}} \mathcal{G}(\chi=0, \alpha) .
\end{aligned}
$$

We can equivalently compute this object by using the equivalence of the canonical and microcanonical ensemble at large $S(E)$,

$$
\begin{aligned}
& \mathcal{G}_{\beta}(\chi, \alpha) \\
& =\frac{1}{Z(\beta)} \operatorname{Tr}\left[e^{-\beta H} \int_{-\infty}^{\infty} \prod_{k} d g_{k} \int_{0}^{\infty} d t \int_{0}^{L} \frac{d s}{L}: e^{\mathcal{F}}:\left(\frac{t}{\pi}\right)^{2}\right]
\end{aligned}
$$

where the "temperature," $\beta^{-1}$, is set by demanding that the expectation value of the Hamiltonian is $N_{1} N_{5}$ and $Z(\beta)=\operatorname{Tr}\left(e^{-\beta H}\right)$. The equivalence of ensembles implies that

$$
\mathcal{G}_{\beta}(\chi, \alpha)=\mathcal{G}(\chi, \alpha)+\mathrm{O}\left(\frac{1}{\sqrt{S(E)}}\right)
$$

and this accuracy is sufficient for our purpose. Similarly, the thermal expectation values, $\left\langle f_{5}\right\rangle_{\beta},\left\langle f_{1}\right\rangle_{\beta}$, and $\left\langle W_{i}\right\rangle_{\beta}$, obtained from this generating function match the typical expectation values of (3.3) up to terms suppressed by the entropy.

Before evaluating the traces we need, we remind the reader of a few simple results. If we consider a single simple harmonic oscillator, corresponding to a given value of $n$ and $k$, then in a number eigenstate of that oscillator, $\left|N_{n}^{k}\right\rangle$, for any values of the c-number coefficients $c_{n}^{k}$ and $d_{n}^{k}$, we have

$$
\begin{aligned}
& \left\langle N_{n}^{k}\left|e^{c_{n}^{k}\left(a_{n}^{k}\right)^{\dagger}} e^{d_{n}^{k} a_{n}^{k}}\right| N_{n}^{k}\right\rangle \\
& =\sum_{r=0}^{N} \frac{N !}{(N-r) !(r !)^{2}}\left(c_{n}^{k} d_{n}^{k}\right)^{r} \\
& =\sum_{r=0}^{\infty} \frac{N_{n}^{k}\left(N_{n}^{k}-1\right) \ldots\left(N_{n}^{k}-r+1\right)}{(r !)^{2}}\left(c_{n}^{k} d_{n}^{k}\right)^{r} .
\end{aligned}
$$

Here, in the last step, we have simply noted that the sum over $r$ can be extended until $\infty$. All terms larger than $N$ vanish because of the factor of $(N-r+1)$ in the numerator. If we take the thermal trace and denote $z=e^{-\beta}$, we find

$$
\begin{aligned}
\operatorname{Tr}\left(e^{-\beta n N_{n}^{k}} e^{c_{n}^{k}\left(a_{n}^{k}\right)^{\dagger}} e^{d_{n}^{k} a_{n}^{k}}\right) & =\sum_{N_{n}^{k}=0}^{\infty} \sum_{r=0}^{\infty} \frac{N_{n}^{k}\left(N_{n}^{k}-1\right) \ldots\left(N_{n}^{k}-r+1\right)}{(r !)^{2}} z^{n N_{n}^{k}}\left(c_{n}^{k} d_{n}^{k}\right)^{r} \\
& =\frac{1}{1-z^{n}} \exp \left(\frac{c_{n}^{k} d_{n}^{k} z^{n}}{1-z^{n}}\right),
\end{aligned}
$$

where we have used the identity

$$
\sum_{N=0}^{\infty} N(N-1) \ldots(N-r+1) x^{N}=r ! \frac{x^{r}}{(1-x)^{r+1}}
$$

We now note that

$$
\mathcal{F}=\sum_{k, n} \frac{\mu e^{-\frac{2 i n n s}{L}} a_{n}^{k}\left(-2 i t g_{k}+\alpha_{k}-i \frac{2 \pi n}{L} \chi_{k}\right)}{\sqrt{2 n} L}+\frac{\mu e^{\frac{2 i \pi n s}{L}}\left(a_{n}^{k}\right)^{\dagger}\left(-2 i t g_{k}+\alpha_{k}+i \frac{2 \pi n}{L} \chi_{k}\right)}{\sqrt{2 n}}-\sum_{k} t_{k}\left(g_{k}-2 i x_{k}\right) .
$$

The expression above is just in the form we need. We see that the thermal trace breaks up into a product of the traces over individual oscillator sectors and, moreover, that for each oscillator the coefficients $c_{n}^{k}$ and $d_{n}^{k}$ can be identified from the expression above. This leads to

$$
\begin{aligned}
\operatorname{Tr}\left(e^{-\beta H}: e^{\mathcal{F}}:\right)= & \exp \left[\sum_{k} 2 i t g_{k} x_{k}-t g_{k}^{2}+\sum_{n} \log \left(1-z^{n}\right)\right. \\
& \left.+\sum_{n} \frac{1}{1-z^{n}}\left(\frac{-2 \mu^{2} t^{2} g_{k}^{2} z^{n}}{n}-\frac{2 i \mu^{2} t g_{k} \alpha_{k} z^{n}}{n}+\frac{2 \pi^{2} \mu^{2} n \chi_{k}^{2} z^{n}}{L^{2}}+\frac{\mu^{2} \alpha_{k}^{2} z^{n}}{2 n}\right)\right] .
\end{aligned}
$$


Note that if we take the limit $t, g_{k}, \alpha_{k}, \chi_{k} \rightarrow 0$ in (3.4) we simply get the partition function, which is

$$
Z(\beta)=e^{\sum_{n, k} \log \left(1-z^{n}\right)} .
$$

We can expand the logarithm in a power series and the interchange the order of sums to get

$$
Z(\beta)=\exp \left[d \sum_{n, m} \frac{1}{m} z^{n m}\right]=\exp \left[d \sum_{m} \frac{z^{m}}{m\left(1-z^{m}\right)}\right] .
$$

At high temperatures, we can approximate

$$
\sum_{m} \frac{z^{m}}{m\left(1-z^{m}\right)}=\sum_{m} \frac{1}{m^{2} \beta}+\mathrm{O}(1)=\frac{\pi^{2}}{6 \beta}(1+\mathrm{O}(\beta)),
$$

and therefore

$$
Z(\beta)=e^{\frac{2 \pi^{2}}{6 \beta}},
$$

where we have dropped the $\mathrm{O}(\beta)$ errors that should be understood and will not be displayed explicitly again.
From the partition function above, we find that the temperature and the energy (at large energies) are related through

$$
E=\frac{2 \pi^{2}}{3 \beta^{2}}
$$

Moreover, the degeneracy of states at a given energy is given by

$$
S(E)=\frac{4 \pi^{2}}{3 \beta}=2 \pi \sqrt{\frac{2 E}{3}}=2 \pi \sqrt{\frac{2 N_{1} N_{5}}{3}} .
$$

To evaluate the expression in (3.4), we need to evaluate one more infinite sum over $n$,

$$
\begin{aligned}
\sum_{n=1}^{\infty} \frac{n e^{-\beta n}}{1-e^{-\beta n}} & =\sum_{n=1, m=1}^{\infty} n e^{-\beta n m} \\
& =\sum_{m=1}^{\infty} \frac{e^{-\beta m}}{\left(1-e^{-\beta m}\right)^{2}} \underset{\beta \rightarrow \infty}{\rightarrow} \frac{\pi^{2}}{6 \beta^{2}}+\mathrm{O}\left(\frac{1}{\beta}\right) .
\end{aligned}
$$

Therefore, we find at "high temperatures" that

$$
\begin{aligned}
\mathcal{G}_{\beta}(\chi, \alpha) & =\int \prod_{k} d g_{k} d t \frac{d s}{L}\left(\frac{t}{\pi}\right)^{2} e^{\sum_{k}\left[-\left(\frac{\pi^{2} \mu^{2} t^{2}}{3 \beta}+t\right) g_{k}^{2}-g_{k}\left(\frac{i \pi^{2} \mu^{2}+\alpha_{k}}{3 \beta}+2 i t x_{k}\right)+\frac{\pi^{2} \mu^{2} \alpha_{k}^{2}}{12 \beta}+\frac{\pi^{4} \mu^{2} x_{k}^{2}}{3 \beta^{2} L^{2}}\right]} \\
& =\int d t \frac{d s}{L} \frac{9 \beta^{2} e^{\left(\frac{\pi^{4} \mu^{2} \vec{x}^{2}}{3 \beta^{2} L^{2}}+\frac{\pi^{2} \vec{\alpha}^{2} \mu^{2}+4 t\left(\pi^{2} \mu^{2} \vec{x} \cdot \vec{\alpha}-3 \beta r^{2}\right)}{4\left(3 \beta+\pi^{2} \mu^{2} t\right)}\right)}}{\left(3 \beta+\pi^{2} \mu^{2} t\right)^{2}} \\
& =\frac{36 \beta^{2} e^{\frac{\pi^{4} \mu^{2} \tilde{x}^{2}}{3 \beta^{2} L^{2}}-\frac{3 \beta r^{2}}{\pi^{2} \mu^{2}}}\left(e^{\frac{\pi^{2} \bar{\alpha}^{2} \mu^{2}}{12 \beta}+\frac{3 \beta r^{2}}{\pi^{2} \mu^{2}}}-e^{\vec{x} \cdot \vec{\alpha}}\right)}{\pi^{4} \vec{\alpha}^{2} \mu^{4}+36 \beta^{2} r^{2}-12 \pi^{2} \beta \mu^{2} \vec{x} \cdot \vec{\alpha}},
\end{aligned}
$$

where $r^{2}=\sum_{k} x^{k} x^{k}$

From this generating function, we can immediately read off the various "thermal" expectation values. We find that

$$
\begin{aligned}
& \left\langle f_{5}-1\right\rangle_{\beta}=Q_{5} \frac{1-e^{-\frac{r^{2}}{\tau}}}{r^{2}} ; \\
& \left\langle f_{1}-1\right\rangle_{\beta}=Q_{5} \frac{24 \tau^{2}\left(1-e^{-\frac{r^{2}}{\tau}}\right)}{\mu^{2} L^{2} r^{2}}=Q_{1} \frac{\left(1-e^{-\frac{r^{2}}{\tau}}\right)}{r^{2}} \\
& \left\langle A_{i}\right\rangle_{\beta}=0 \\
& \left\langle W_{i}\right\rangle_{\beta}=-Q_{5} \frac{\tau x_{i} e^{-\frac{r^{2}}{\tau}}\left(1-e^{\frac{r^{2}}{\tau}}+\frac{r^{2}}{\tau}\right)}{r^{4}}
\end{aligned}
$$

where

$$
\tau=\frac{\pi^{2} \mu^{2}}{3 \beta}
$$

In the second line of (3.6), we noted that

$$
\frac{24 \tau^{2}}{\mu^{2} L^{2}}=\frac{8 \pi^{4} \mu^{2}}{3 \beta^{2} L^{2}}=\frac{Q_{1}}{Q_{5}}
$$

As advertised, $\left\langle A_{i}\right\rangle_{\beta}$ vanishes in the average fuzzball metric.

\section{Analysis of one-point functions}

The expressions in (3.6) start deviating from the conventional expressions when $r^{2}=\tau$. To understand this physically, we consider the radius of the $y$ circle when $r^{2}=\tau$ in the conventional metric. We see that this radius (in the Einstein frame) is given by 


$$
R_{\text {stretch }}^{2}=\left(\frac{Q_{5}}{Q_{1}}\right)^{\frac{1}{4}}\left(\frac{1}{Q_{1} Q_{5}}\right)^{\frac{1}{2}} \tau R^{2}
$$

Note that we have

$$
\tau=\frac{\mu^{2} \pi^{2}}{3 \beta}=\frac{\mu^{2} S(E)}{4}=\frac{g_{s}^{2} S(E)}{4 R^{2} V_{4}},
$$

where we used the fact that $\mu^{2}=\frac{g_{s}^{2}}{R^{2} V_{4}}$. We also note that the volume of the compact manifold in the string frame is given by $V_{\text {com }}=\left(\frac{Q_{1}}{Q_{5}}\right) V_{4}$.

Putting all these factors together, and using (3.2) and (3.5), we find

$$
\begin{aligned}
R_{\text {stretch }}^{2} & =\left(\frac{Q_{5}}{Q_{1}}\right)^{\frac{1}{4}}\left(\frac{V_{4}}{g_{s}^{2} N_{1} N_{5}}\right)^{\frac{1}{2} g_{s}^{2} 2 \pi \sqrt{\frac{2 N_{1} N_{5}}{3}}} \\
& =\left(\frac{Q_{1}}{Q_{5}}\right)^{\frac{1}{4}} \frac{\pi}{2} \sqrt{\frac{2}{3}} \frac{g_{s}}{\sqrt{V_{\text {com }}}} .
\end{aligned}
$$

In these units, in which the string scale is set to unity, the fundamental (ten-dimensional) Planck scale is simply given by $\ell_{\mathrm{pl}}^{8}=g_{s}^{2}$. Therefore, we have found that

$$
R_{\text {stretch }}^{2}=\frac{\pi}{2} \sqrt{\frac{2}{3}}\left(\frac{Q_{1}}{Q_{5}}\right)^{\frac{1}{4}} \frac{\ell_{\mathrm{pl}}^{4}}{\sqrt{V_{\mathrm{com}}}} .
$$

Now, we should work in the duality frame, in which the physical volume of the compact manifold is at least $V_{\text {com }} \geq$ 1 in string units. If we are not in such a frame, we should use T-duality in the compact directions to reach such a frame. Moreover, we should work in the duality frame in which the dilaton does not blow up at this point in space, and therefore we need $\frac{Q_{1}}{Q_{5}}=\mathrm{O}(1)$. Just as above, if this constraint is not met, we can use the U-duality group to change the values of $N_{1}$ and $N_{5}$ while keeping $N_{1} N_{5}$ constant to reach such a duality frame. Putting these physical constraints into (3.8), we see that

$$
R_{\text {stretch }} \ll \mathrm{O}\left(\ell_{\mathrm{pl}}\right) \text {. }
$$

Therefore, in the average fuzzball solution, the metric starts to differ from the conventional metric when the $y$ circle has a size that is smaller than the Planck length. The solution is completely unreliable since, well before this size is reached, classical string effects that have not been taken into account in obtaining the solution (3.1) become important. Moreover, in this region, as one might expect-and as we compute explicitly in the next subsection-quantum fluctuations in the geometry are as large as various classical expectation values. This implies that we should not take (3.1) with the substitutions (3.6) seriously as a quantumcorrected geometry.

$$
\begin{gathered}
\text { B. Fluctuations: } \\
\left\langle\Psi\left|\left(f_{5}-\mathbf{1}\right)^{2}\right| \Psi\right\rangle,\left\langle\Psi\left|\left(f_{1}-\mathbf{1}\right)^{2}\right| \Psi\right\rangle,\left\langle\Psi\left|W_{i} W_{j}\right| \Psi\right\rangle
\end{gathered}
$$

We now compute the quantum fluctuations in the thermally averaged ensemble as an input to computing the quantumness parameters for these quantities. We do not compute these parameters for $A_{i}$, since it vanishes in both the conventional geometry and the black hole. We consider the generating function of the variance

$$
\mathcal{V}_{\beta}(\chi, \alpha, \tilde{\chi}, \tilde{\alpha})=\frac{1}{Z(\beta)} \operatorname{Tr}\left[e^{-\beta H} \int_{-\infty}^{\infty} \prod_{k} d g_{k} d \tilde{g}_{k} \int_{0}^{\infty} d t d \tilde{t} \int_{0}^{L} \frac{d s d \tilde{s}}{L^{2}}\left(\frac{\tilde{t} \tilde{t}}{\pi^{2}}\right)^{2}: e^{\mathcal{F}}:: e^{\tilde{\mathcal{F}}}:\right]
$$

where

$$
\tilde{\mathcal{F}}=-\sum_{k} \tilde{t} \tilde{g}_{k} \tilde{g}_{k}+2 i \tilde{t}\left(x^{k}-F^{k}(\tilde{s})\right) \tilde{g}_{k}+\sum_{j} \tilde{\alpha}^{j} F^{j}(\tilde{s})+\tilde{\chi}^{j} \frac{d F^{j}(\tilde{s})}{d \tilde{s}} .
$$

(Note that $\mathcal{F}$ and $\tilde{\mathcal{F}}$ share the same value of $x$.) We can then obtain the required two-point functions by differentiation:

$$
\begin{aligned}
\left\langle\left(f_{5}-1\right)^{2}\right\rangle_{\beta} & =Q_{5}^{2} \mathcal{V}_{\beta}(\chi=0, \tilde{\chi}=0, \alpha=0, \tilde{\alpha}=0) ; \\
\left\langle\left(f_{1}-1\right)^{2}\right\rangle_{\beta} & =Q_{5}^{2} \lim _{\chi^{i}, \tilde{\chi}^{i} \rightarrow 0} \sum_{i, j} \frac{\partial^{4}}{\partial^{2} \chi^{i} \partial^{2} \tilde{\chi}^{i}} \mathcal{V}_{\beta}(\chi, \tilde{\chi}, \alpha=0, \tilde{\alpha}=0) ; \\
\left\langle W_{i} W_{j}\right\rangle_{\beta} & =Q_{5}^{2} \lim _{\alpha^{i}, \tilde{\alpha}^{i} \rightarrow 0} \frac{\partial^{2}}{\partial \alpha_{i} \partial \tilde{\alpha}_{j}} \mathcal{V}_{\beta}(\chi=0, \alpha, \tilde{\chi}=0, \tilde{\alpha}) .
\end{aligned}
$$

By the equivalence of ensembles, these variances coincide with microcanonical variances up to terms suppressed by the entropy. Then, by Result 1, these variances also coincide with variances computed in a typical state,

$$
\left\langle\left(f_{5}-1\right)^{2}\right\rangle_{\beta}=\left\langle\Psi\left|\left(f_{5}-1\right)^{2}\right| \Psi\right\rangle+\mathrm{O}\left(\frac{1}{\sqrt{S(E)}}\right)
$$

and similarly for $\left\langle\left(f_{1}-1\right)^{2}\right\rangle_{\beta}$ and $\left\langle W_{i} W_{j}\right\rangle_{\beta}$. 
The computation of the variance is considerably more involved than the computation of the expectation value. So, we will not compute the function $\mathcal{V}_{\beta}$ for arbitrary values of its parameters but simply compute the derivatives and limits in which we are interested.

First, we note that

$$
: e^{\mathcal{F}}:: e^{\tilde{\mathcal{F}}}:=: e^{\mathcal{F}+\tilde{\mathcal{F}}}: e^{\mathcal{N}},
$$

where the normal-ordering constant $\mathcal{N}$ arises because we need to move the creation operators inside $\tilde{\mathcal{F}}$ past the annihilation operators of $\mathcal{F}$. This can be easily done through the formula

In our case, we find that

$$
\begin{aligned}
\mathcal{N}= & \sum_{n, k} e^{\frac{2 \pi i n(\tilde{s}-s)}{L}}\left[\frac{1}{n}\left(-2 \mu^{2} \tilde{t} \tilde{g}_{k} \tilde{g}_{k}-i \mu^{2} \operatorname{tg}_{k} \tilde{\alpha}_{k}-i \mu^{2} \tilde{t} \tilde{g}_{k} \alpha_{k}+\frac{1}{2} \mu^{2} \alpha_{k} \tilde{\alpha}_{k}\right)\right. \\
& \left.+\left(\frac{2 \pi \mu^{2} t g_{k} \tilde{\chi}_{k}}{L}-\frac{2 \pi \mu^{2} \tilde{t} \tilde{g}_{k} \chi_{k}}{L}+\frac{i \pi \mu^{2} \alpha_{k} \tilde{\chi}_{k}}{L}-\frac{i \pi \mu^{2} \tilde{\alpha}_{k} \chi_{k}}{L}\right)+\frac{2 \pi^{2} n \mu^{2} \chi_{k} \tilde{\chi}_{k}}{L^{2}}\right] \\
= & {\left[-\log \left(1-e^{\frac{2 \pi i(\tilde{s}-s)}{L}}\right)\left(-2 \mu^{2} t \tilde{t} g_{k} \tilde{g}_{k}-i \mu^{2} t g_{k} \tilde{\alpha}_{k}-i \mu^{2} \tilde{t} \tilde{g}_{k} \alpha_{k}+\frac{1}{2} \mu^{2} \alpha_{k} \tilde{\alpha}_{k}\right)\right.} \\
& +\frac{1}{\left(1-e^{\left.\frac{2 \pi i(\tilde{s}-s)}{L}\right)}\right.}\left(\frac{2 \pi \mu^{2} t g_{k} \tilde{\chi}_{k}}{L}-\frac{2 \pi \mu^{2} \tilde{t} \tilde{g}_{k} \chi_{k}}{L}+\frac{i \pi \mu^{2} \alpha_{k} \tilde{\chi}_{k}}{L}-\frac{i \pi \mu^{2} \tilde{\alpha}_{k} \chi_{k}}{L}\right)+\frac{1}{\left(1-e^{\left.\frac{2 \pi i(\tilde{s}-s)}{L}\right)^{2}} \frac{2 \pi^{2} \mu^{2} \chi_{k} \tilde{\chi}_{k}}{L^{2}}\right] .}
\end{aligned}
$$

Also,

$$
\begin{aligned}
\mathcal{F}+\tilde{\mathcal{F}}= & \sum_{n, k} \frac{1}{2 L \sqrt{n}}\left[\sqrt{2} \mu a_{n}^{k}\left(e^{-\frac{2 i \pi n \tilde{s}}{L}}\left(-2 i L \operatorname{tg} g_{k}+L \alpha_{k}-2 i \pi n \chi_{k}\right)+e^{-\frac{2 i \pi n n s}{L}}\left(-2 i L \tilde{t} \tilde{g}_{k}+L \tilde{\alpha}_{k}-2 i \pi n \tilde{\chi}_{k}\right)\right)\right. \\
& +\sqrt{2} \mu\left(a_{n}^{k}\right)^{\dagger}\left(e^{\frac{2 i n n s}{L}}\left(-2 i L t g_{k}+L \alpha_{k}+2 i \pi n \chi_{k}\right)+e^{\frac{2 i \pi n \tilde{s}}{L}}\left(-2 i L \tilde{t} \tilde{g}_{k}+L \tilde{\alpha}_{k}+2 i \pi n \tilde{\chi}_{k}\right)\right) \\
& \left.-2 L \sqrt{n}\left(-2 i x_{k}\left(t g_{k}+\tilde{t} \tilde{g}_{k}\right)+t g_{k}^{2}+\tilde{t} \tilde{g}_{k}^{2}\right)\right] .
\end{aligned}
$$

Therefore,

$$
\left\langle: e^{\mathcal{F}+\tilde{\mathcal{F}}}:\right\rangle_{\beta}=e^{\sum_{n, k} \mathcal{Q}_{n, k}},
$$

with

$$
\begin{aligned}
\mathcal{Q}_{n, k}= & \frac{\mu^{2} z^{n}}{2 n\left(z^{n}-1\right)}\left(2 \operatorname{tg}_{k} e^{\frac{2 i \pi n \tilde{s}}{L}}+2 \tilde{t} \tilde{g}_{k} e^{\frac{2 i \pi n s}{L}}+i \tilde{\alpha}_{k} e^{\frac{2 i \pi n s}{L}}+\frac{2 \pi n}{L} \tilde{\chi}_{k} e^{\frac{2 i \pi n n s}{L}}+i \alpha_{k} e^{\frac{2 i \pi n \tilde{s}}{L}}+\frac{2 \pi n}{L} \chi_{k} e^{\frac{2 i \pi n \tilde{s}}{L}}\right) \\
& \times\left(2 t g_{k} e^{\frac{-2 i \pi n s}{L}}+2 \tilde{t} \tilde{g}_{k} e^{\frac{-2 i \pi n \tilde{s}}{L}}+i \alpha_{k} e^{\frac{-2 i \pi n s}{L}}-\frac{2 \pi n}{L} \chi_{k} e^{\frac{-2 i \pi n n s}{L}}+i \tilde{\alpha}_{k} e^{\frac{-2 i \pi n \tilde{s}}{L}}-\frac{2 \pi n}{L} \tilde{\chi}_{k} e^{\frac{-2 i \pi n \tilde{s}}{L}}\right) \\
& -\operatorname{tg}_{k}\left(g_{k}-2 i x_{k}\right)-\tilde{t} \tilde{g}_{k}\left(\tilde{g}_{k}-2 i x_{k}\right) .
\end{aligned}
$$

We can compute the sums over $n$ using the following formulas. As $\beta \rightarrow 0$, we have

$$
\begin{aligned}
\sum_{n} \frac{e^{-\beta n+2 i \pi n s}}{n\left(1-e^{-n \beta}\right)} & \rightarrow \frac{\mathrm{Li}_{2}\left(e^{2 i \pi s}\right)}{\beta} ; \\
\sum_{n} \frac{e^{-\beta n+2 i \pi n s}}{1-e^{-n \beta}} & \rightarrow-\frac{\log \left(1-e^{2 i \pi s}\right)}{\beta} ; \\
\sum_{n} \frac{n e^{-\beta n+2 i \pi n s}}{1-e^{-n \beta}} & \rightarrow \frac{1}{\beta\left(1-e^{2 i \pi s}\right)} .
\end{aligned}
$$


We can then write

$$
\mathcal{N}+\sum_{n, k} \mathcal{Q}_{n, k}=\sum_{k}-\left(g^{k}, \tilde{g}^{k}\right) \cdot\left(\begin{array}{cc}
\tau t^{2}+t & c t \tilde{t} \\
c t \tilde{t} & \tau \tilde{t}^{2}+\tilde{t}
\end{array}\right) \cdot\left(g^{k}, \tilde{g}^{k}\right)+\ell_{1}^{k} t g^{k}+\ell_{2}^{k} \tilde{g}^{k}+z
$$

where

$$
\tau=\frac{\pi^{2} \mu^{2}}{3 \beta}
$$

as above in Sec. III A, and the other coefficients take on the values

$$
\begin{aligned}
c & =\frac{\mu^{2}}{\beta} \mathcal{L}^{+}+c_{\mathcal{N}} ; \\
-i \ell_{1}^{k} & =2 x_{k}-\frac{\mu^{2}}{3 \beta}\left[\pi^{2} \alpha_{k}+3 \tilde{\alpha}_{k} \mathcal{L}^{+}-6 i \pi \frac{\tilde{\chi}_{k}}{L} \mathcal{L}^{-}\right]-i \ell_{1 \mathcal{N}}^{k} ; \\
-i \ell_{2}^{k} & =2 x_{k}-\frac{\mu^{2}}{3 \beta}\left[\pi^{2}(\tilde{\alpha})_{k}+3 \alpha_{k} \mathcal{L}^{+}+6 i \pi \frac{\chi_{k}}{L} \mathcal{L}^{-}\right]-i \ell_{2 \mathcal{N}}^{k} ; \\
z & =\frac{\mu^{2}}{12 \beta^{2}}\left[\pi^{2} \beta \alpha_{k}^{2}+\left(-24 \pi^{2} \beta \frac{\chi_{k} \tilde{\chi}_{k}}{L^{2}}+4 \pi^{4} \frac{\chi_{k}^{2}}{L^{2}}+4 \pi^{4} \frac{\tilde{\chi}_{k}^{2}}{L^{2}}\right)+\pi^{2} \beta \tilde{\alpha}_{k}^{2}+12 i \pi \beta \tilde{\alpha}_{k} \frac{\chi_{k}}{L} \mathcal{L}^{-}+\alpha_{k} \tilde{\alpha}_{k} \mathcal{L}^{+}-12 i \pi \beta \frac{\tilde{\chi}_{k}}{L} \mathcal{L}^{-}\right]+z_{\mathcal{N}},
\end{aligned}
$$

where

$$
\begin{aligned}
& \mathcal{L}^{+}=L i_{2}\left(e^{-\frac{2 i \pi(s-\tilde{s})}{L}}\right)+L i_{2}\left(e^{\frac{2 i \pi(s-\tilde{s})}{L}}\right) \\
& \mathcal{L}^{-}=\log \left(1-e^{-\frac{2 i \pi(s-\tilde{s})}{L}}\right)-\log \left(1-e^{\frac{2 i \pi(s-\tilde{s})}{L}}\right) .
\end{aligned}
$$

The contribution from the normal-ordering constant in the coefficients above is indicated with the subscript $\mathcal{N}$, and these numbers are given by

$$
\begin{aligned}
& c_{\mathcal{N}}=\mu^{2} \mathcal{L}_{\mathcal{N}} \\
& \ell_{1 \mathcal{N}}=-i \mu^{2}\left(i \tilde{\alpha}_{k} \mathcal{L}_{\mathcal{N}}+\frac{2 \pi \tilde{\chi}_{k}}{L(-1+w)}\right) \\
& \ell_{2 \mathcal{N}}=-i \mu^{2}\left(i \alpha_{k} \mathcal{L}_{\mathcal{N}}-\frac{2 \pi \chi_{k}}{L(-1+w)}\right) \\
& z_{\mathcal{N}}=-\frac{\mu^{2}}{2(-1+w)^{2}}\left[\alpha_{k}(-1+w)\left(\tilde{\alpha}_{k}(-1+w) \mathcal{L}_{\mathcal{N}}-2 i \pi \frac{\tilde{\chi}_{k}}{L}\right)-2 \pi \frac{\chi_{k}}{L}\left(2 \pi \frac{\tilde{\chi}_{k}}{L} w-i \tilde{\alpha}_{k}(-1+w)\right)\right]
\end{aligned}
$$

with $w=e^{\frac{2 i \pi(s-\tilde{s})}{L}}$ and $\mathcal{L}_{\mathcal{N}}=\log (1-1 / w)$. By comparing (3.10) to (3.9), we see that the coefficients that come from normal ordering are all negligible when $\beta \ll 1$. So, for numerical purposes, we will neglect these coefficients, although the fact that they are nonzero will play a role below.

In this form, we can immediately do the integral over $g_{k}$ and $\tilde{g}_{k}$, since these are Gaussian. We find that

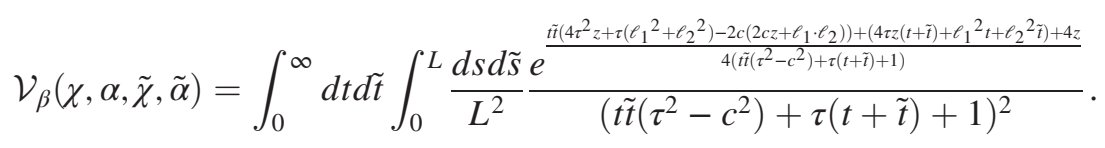

At this stage, rather than doing the $t$ integrals in generality, it is convenient to separate the computation of the different quantities. 


\section{1. $\left\langle\left(f_{5}-1\right)^{2}\right\rangle_{\beta}$}

This is the simplest $t$ integral to calculate since we simply set $\chi, \tilde{\chi}, \alpha, \tilde{\alpha}$ to 0 . With these substitutions, $z \rightarrow 0$, and also $\ell_{1}^{k}, \ell_{2}^{k} \rightarrow 2 i x^{k}$. We then find that

$$
\begin{aligned}
\mathcal{V}_{\beta}(\chi=0, \alpha=0, \tilde{\chi}=0, \tilde{\alpha}=0)= & \int_{0}^{L} \frac{d s}{L} \frac{d \tilde{s}}{L} \int_{0}^{\infty} d t d \tilde{t} \frac{\exp \left(-\frac{r^{2}(2 \tilde{t}(\tau-c)+(t+\tilde{t}))}{\tilde{t}\left(\tau^{2}-c^{2}\right)+\tau(t+\tilde{t})+1}\right)}{\left(\tilde{t} \tilde{t}\left(\tau^{2}-c^{2}\right)+\tau(t+\tilde{t})+1\right)^{2}} \\
= & \int_{0}^{L} \frac{d s}{L} \frac{d \tilde{s}}{L}\left[\frac{e^{-\frac{r^{2}}{c}}}{c^{2}}\left(\operatorname{Ei}\left(\frac{r^{2}}{c}\right)-2 \operatorname{Ei}\left(\frac{(\tau-c) r^{2}}{\tau c}\right)+\operatorname{Ei}\left(\frac{(\tau-c) r^{2}}{c(\tau+c)}\right)\right)\right. \\
& \left.+\frac{2 \tau e^{-\frac{r^{2}}{\tau}}}{c r^{2}(\tau-c)}-\frac{(\tau+c) e^{-\frac{2 r^{2}}{\tau+c}}}{c r^{2}(\tau-c)}-\frac{1}{c r^{2}}\right] .
\end{aligned}
$$

Here, $E i(x)=-\int_{-x}^{\infty} e^{-t} \mathcal{P}\left(\frac{1}{t}\right) d t$. The last integral, over $s, \tilde{s}$ must be done numerically, and we discuss that in Sec. III. C. 1.

\section{2. $\left\langle\left(f_{1}-1\right)^{2}\right\rangle_{\beta}$}

It is convenient to first differentiate with respect to $\chi, \tilde{\chi}$ before performing the $t, \tilde{t}$ integrals. These derivatives lead to a complicated expression. However, it is important to realize that this expression involves terms that appear at different orders in $\frac{1}{\beta}$.

The reader can persuade herself through inspection, or through an explicit calculation, that the dominant terms at small $\beta$ occur only when the $\chi, \tilde{\chi}$ derivatives act on the $\chi_{k}^{2}$ and $\left(\tilde{\chi}^{k}\right)^{2}$ terms inside $z$ in (3.9).

This leads to the simple result

$$
\left\langle\left(f_{1}-1\right)^{2}\right\rangle_{\beta}=\frac{64 \pi^{8} \mu^{4}}{9 L^{4} \beta^{4}}\left\langle\left(f_{5}-1\right)^{2}\right\rangle_{\beta}(1+\mathrm{O}(\beta))=\frac{Q_{1}^{2}}{Q_{5}^{2}}\left\langle\left(f_{5}-1\right)^{2}\right\rangle_{\beta}(1+\mathrm{O}(\beta)),
$$

where we have used the ratio between coefficients displayed in (3.7) and neglected the $O(\beta)$ terms in the last equality.

$$
\text { 3. }\left\langle W_{i} W_{j}\right\rangle_{\beta}
$$

The computation of $\left\langle W_{i} W_{j}\right\rangle_{\beta}$ is rather involved. We do not give the details of all the intermediate steps but simply note the final answer in the form of an integral over $s, \tilde{s}$ that we will evaluate numerically below. We find that

$$
\frac{1}{Q_{5}^{2}}\left\langle W_{i} W_{j}\right\rangle_{\beta}=\mathcal{A} \delta_{i j}+\mathcal{B} x_{i} x_{j},
$$

where

$$
\begin{aligned}
\mathcal{A}= & \frac{(c-\tau) e^{-\frac{r^{2}}{c}}\left(r^{2}(c-\tau)+3 c(\tau+c)\right)\left(-2 \operatorname{Ei}\left(\frac{(\tau-c) r^{2}}{\tau c}\right)+\operatorname{Ei}\left(\frac{(\tau-c) r^{2}}{c(\tau+c)}\right)+\operatorname{Ei}\left(\frac{r^{2}}{c}\right)\right)}{12 c^{4}} \\
& +\frac{\tau e^{-\frac{r^{2}}{\tau}}\left(-\tau c^{2}(\tau+3 c)+r^{4}(\tau-c)^{2}+c r^{2}(c-\tau)(2 \tau+3 c)\right)}{6 c^{3} r^{4}(\tau-c)} \\
& +\frac{(\tau+c) e^{-\frac{2 r^{2}}{\tau+c}}\left(c^{2} r^{2}(\tau+c)^{2}+r^{6}\left(-(\tau-c)^{2}\right)+2 c r^{4}(\tau-c)(\tau+c)\right)}{12 c^{3} r^{6}(\tau-c)},
\end{aligned}
$$


and

$$
\begin{aligned}
\mathcal{B}= & \frac{\left(\tau^{2}+4 \tau c+c^{2}\right) e^{-\frac{r^{2}}{c}}\left(-2 \operatorname{Ei}\left(\frac{(\tau-c) r^{2}}{\tau c}\right)+\operatorname{Ei}\left(\frac{(\tau-c) r^{2}}{c(\tau+c)}\right)+\operatorname{Ei}\left(\frac{r^{2}}{c}\right)\right)}{6 c^{4}} \\
& +\frac{\tau e^{-\frac{r^{2}}{\tau}}\left(\tau^{2}\left(2 c^{2}+c r^{2}+r^{4}\right)+\tau c\left(6 c^{2}+5 c r^{2}+4 r^{4}\right)+c^{2} r^{2}\left(6 c+r^{2}\right)\right)}{3 c^{3} r^{6}(\tau-c)} \\
& -\frac{(\tau+c) e^{-\frac{2 r^{2}}{\tau+c}}\left(\tau^{2}\left(2 c^{2}+c r^{2}+r^{4}\right)+2 \tau c\left(2 c^{2}+3 c r^{2}+2 r^{4}\right)+c^{2}\left(2 c^{2}+5 c r^{2}+r^{4}\right)\right)}{6 c^{3} r^{6}(\tau-c)} .
\end{aligned}
$$

\section{Analysis of the results}

We now proceed to analyze the results obtained. We will compute the difference and quantumness parameters defined in (2.5) and (2.6). For the harmonic functions, we compute the following expressions, both of which depend on $\vec{x}$ :

$$
\mathrm{d}_{5}=\left|\frac{\left\langle\left(f_{5}-1\right)\right\rangle_{\beta}-f_{5}^{\mathrm{bh}}+1}{\left\langle\left(f_{5}-1\right)\right\rangle_{\beta}}\right| ; \quad \mathrm{q}_{5}=\left|\frac{\left(\left\langle\left(f_{5}-1\right)^{2}\right\rangle_{\beta}-\left\langle\left(f_{5}-1\right)\right\rangle_{\beta}^{2}\right)^{\frac{1}{2}}}{\left\langle\left(f_{5}-1\right)\right\rangle_{\beta}}\right| .
$$

We do not need to compute these parameters separately for $f_{1}$ since, as we have found above, $\left\langle f_{1}-1\right\rangle_{\beta}=\frac{Q_{1}}{Q_{5}}\left\langle f_{5}-1\right\rangle_{\beta}$ and also $\left\langle\left(f_{1}-1\right)^{2}\right\rangle_{\beta}=\frac{Q_{1}^{2}}{Q_{5}^{2}}\left\langle\left(f_{5}-1\right)^{2}\right\rangle_{\beta}$. Therefore, $\mathrm{q}_{1}=\mathrm{q}_{5} ; \mathrm{d}_{1}=\mathrm{d}_{5}$.

Since $W_{i}=0$ in the conventional black-hole geometry, we find $d_{W}=1$. Then, to measure the size of quantum fluctuations, we compute

$$
\mathbf{q}_{W}=\left|\frac{\left(x^{i} x^{j}\left\langle W_{i} W_{j}\right\rangle_{\beta}-x^{i} x^{j}\left\langle W_{i}\right\rangle_{\beta}\left\langle W_{j}\right\rangle_{\beta}\right)^{\frac{1}{2}}}{x^{i} x^{j}\left\langle W_{i}\right\rangle_{\beta}\left\langle W_{j}\right\rangle_{\beta}}\right| .
$$

\section{Difference and quantumness parameters for the harmonic functions, $f_{1}$ and $f_{5}$}

We start by evaluating (3.14). Let us consider the remaining integrals over $s, \tilde{s}$ in the results (3.11).

First, note that $s, \tilde{s}$ only appear in these expressions through $w=e^{\frac{2 \pi i(s-5)}{L}}$. Moreover, each integrand has a Laurent series expansion in $w$, and so the integral over $s, \tilde{s}$ simply picks out the $w^{0}$ term. Note also that the integrand has potential singularities at $\tau=c$. These poles arise when $w$ solves

$$
\frac{\pi^{2}}{6}-\frac{1}{2}\left(\mathrm{Pl}(2, w)+\mathrm{Pl}(2,1 / w)+\frac{2 \pi \beta}{L} \log (1-w)\right)=0 .
$$

At small $\beta$, the pole is almost at $w=1$, but the last term moves the pole slightly off the $|w|=1$ contour to $|w|=1+\mathrm{O}(\beta)$. Other than the effect above, the normalordering term is negligible. For ease with the final numerical integrals, we deal with this as follows. We drop the term $c_{\mathcal{N}}$ in (3.9) and instead add a small real regulator to $c$ so that $c_{\epsilon}=c-\epsilon$. We then compute (3.11) and (3.13) with $c \rightarrow c_{\epsilon}$. Numerically, it is easy to check that the value of this regulator does not alter any of the answers, provided it is kept small enough.

It is of interest to consider the behavior near $r=0$, where the fuzzball solution differs from the conventional solution. We find that, in this limit (3.11),

$$
\begin{aligned}
\frac{1}{Q_{5}^{2}}\left\langle\left(f_{5}-1\right)^{2}\right\rangle_{\beta}= & Q_{5}^{2} \int_{0}^{L} \frac{d s d \tilde{s} \log \left(\frac{\tau^{2}}{\tau^{2}-c^{2}}\right)}{c^{2}} \\
& +\frac{r^{2}\left(\tau(\tau+c) \log \left(1-\frac{c^{2}}{\tau^{2}}\right)+c^{2}\right)}{\tau c^{3}(\tau+c)}+O\left(r^{4}\right) \\
= & 1.182 \frac{1}{\tau^{2}}-1.283 \frac{r^{2}}{\tau^{3}}
\end{aligned}
$$

which leads to

$$
\mathrm{q}_{5}=0.426-0.119 \frac{r^{2}}{\tau}
$$

They key point is that, just as expected, $\mathrm{q}_{5}$ becomes of order 1 just in the region where $\frac{r^{2}}{\tau} \ll 1$ and the average fuzzball geometry starts to differ appreciably from the conventional solution. Therefore, in precisely the region where the average fuzzball geometry predicts interesting effects, it becomes unreliable.

It is possible to numerically compute both $d_{5}$ and $q_{5}$ for larger values of $x$, and we display this in Fig. 5. As we move to larger values of $\frac{r^{2}}{\tau}$, the solution becomes more 


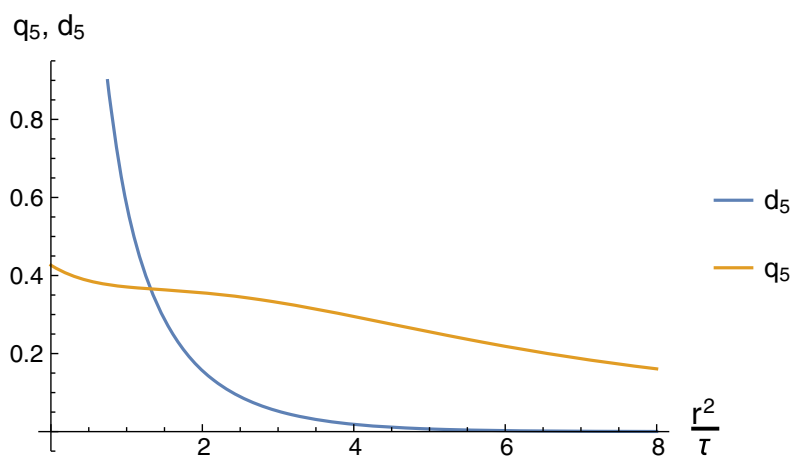

FIG. 5. A plot of the difference parameter, $d_{5}$, and the "quantumness parameter", $\mathrm{q}_{5}$, for the harmonic functions $f_{5}$ and $f_{1}$. The plot shows that in the average fuzzball geometry, precisely in the region where $f_{5}$ and $f_{1}$ differ significantly from their conventional value, quantum fluctuations becomes large and the fuzzball geometry becomes unreliable.

reliable, but then it also becomes indistinguishable from the conventional black hole.

\section{Difference and quantumness parameters for $W_{i}$}

We now turn to the difference and quantumness parameters for $W_{i}$. The difference parameter is uniformly equal to 1 since $W_{i}=0$ in the conventional black hole.

At small $\frac{r^{2}}{\tau}$, we find that

$$
\begin{aligned}
\hat{x}^{i} \hat{x}^{j}\left\langle W_{i} W_{j}\right\rangle_{\beta}= & \int_{0}^{L} \frac{d s}{L} \frac{d \tilde{s}}{L}\left[\frac{\left(\tau^{2}-c^{2}\right) \log \left(\frac{\tau^{2}-c^{2}}{\tau^{2}}\right)+c^{2}}{4 c^{3}}\right. \\
& \left.-\frac{r^{2}(\tau+c)\left(\tau^{2} \log \left(\frac{\tau^{2}-c^{2}}{\tau^{2}}\right)+c^{2}\right)}{2 \tau c^{4}}\right]+\mathrm{O}\left(\frac{r^{4}}{\tau^{3}}\right) \\
= & \frac{0.00489}{\tau}+\frac{0.355 r^{2}}{\tau^{2}}+\mathrm{O}\left(\frac{r^{4}}{\tau^{3}}\right) .
\end{aligned}
$$

At small values of $r$, when we combine this with the series expansion of (3.6), this leads to

$$
\mathrm{q}_{W}=0.140 \frac{\sqrt{\tau}}{r}+1.587 \frac{r}{\sqrt{\tau}} .
$$

Therefore, quantum fluctuations diverge near $r=0$, and the expected value of $W_{i}$ in the average geometry becomes completely unreliable.

We can numerically plot the quantumness parameter for larger values of $r$, and this leads to the curve shown in Fig. 6. At both small $\frac{r^{2}}{\tau}$ and larger values of $\frac{r^{2}}{\tau}$, the value for $W_{i}$ given by the average fuzzball geometry is unreliable.

\section{Fuzzballs and entropy counting}

In this section, we have argued that the average fuzzball geometry is unreliable in the interesting region near $r=0$. By Result 1, the geometry corresponding to a typical state

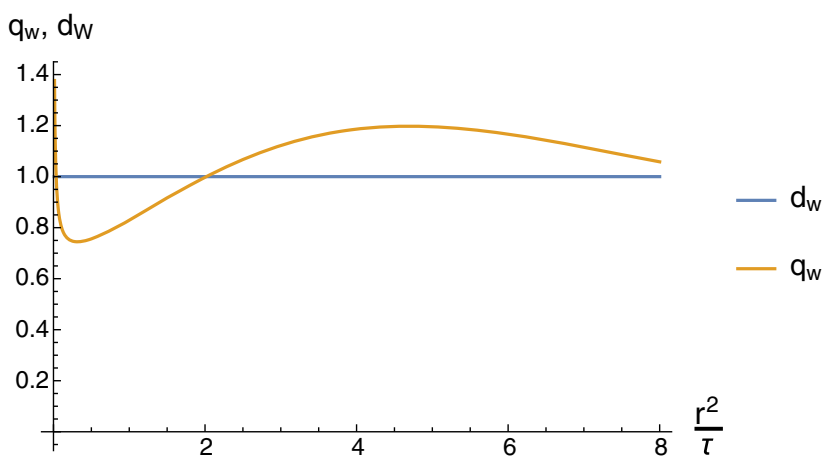

FIG. 6. A plot of the difference parameter, $d_{W}$, and the quantumness parameter, $\mathrm{q}_{W}$, for the 1 -form $W_{i}$. The difference parameter is uniformly 1 , since this function vanishes in the conventional black hole. But the value it ostensibly takes in the average fuzzball solution is always unreliable since quantum fluctuations are the same size as its expectation value.

in the Hilbert space produced by quantizing fuzzball solutions is also unreliable near $r=0$. Away from $r=0$, this average geometry is effectively indistinguishable from the conventional geometry since its deviation from the conventional geometry is of the same order as quantum fluctuations.

The reader might wonder how-in spite of this factcounting the entropy of fuzzball solutions succeeds in getting the correct form for the number of Ramond ground states in the D1-D5 system.

The puzzle is made more acute by recognizing that most of the contribution to this entropy comes from Planck-size fuzzballs. For small $\beta$, the reader can check that $\tau$ also measures the average size of the profile function

$$
\sum_{i} \int_{0}^{L}\left\langle: F^{i}(s) F^{i}(s):\right\rangle_{\beta} \frac{d s}{L} .=2 \tau .
$$

Fluctuations in the size of the fuzzball are also controlled by $\tau$. At high temperatures,

$$
\sum_{i, j} \int_{0}^{L}\left\langle: F^{i}(s) F^{i}(s):: F^{j}(\tilde{s}) F^{j}(\tilde{s}):\right\rangle_{\beta} \frac{d s}{L} \frac{d \tilde{s}}{L}=\frac{22}{5} \tau^{2} .
$$

This tells us that if we consider the entropy corresponding to fuzzballs that are parametrically larger than (3.15) then this entropy is highly suppressed.

We do not have a complete explanation for the fact that quantizing the space of fuzzballs gives approximately the correct counting - even though individual fuzzball solutions that contribute dominantly to the entropy are unreliable. Our best guess is as follows. We can consider the solutions (3.1) for profile functions, $F^{i}(s)$, that are parametrically larger than (3.15). In this regime, the solutions are reliable. Since they also saturate the BPS condition, they must correspond to some ground states of the D1-D5 system. This subclass of solutions can be quantized and counted reliably. Then-perhaps as a result of one of the 
fortuitous coincidences that occur while counting supersymmetric states - this counting formula can be extrapolated to obtain a count of all ground states. Perhaps this last step can be explained by going to some other point in parameter space, where these solutions can be mapped to better controlled states; this deserves to be understood better.

However, we emphasize that the entropy formula itself cannot be taken as evidence that fuzzballs are giving us an accurate picture of physics near $r=0$ at the supergravity point in moduli space, since the solutions that dominate the entropy are unreliable in that region. It would be nice to understand the physics near $r=0$ better, but this clearly requires some other technique.

Another interesting open question is as follows. What is the basis of which the elements minimize $q_{5}, q_{1}$, and $q_{W}$ so that the fluctuations in a typical state-as calculated in (3.11), (3.12), and (3.13)—come from differences between elements of this basis rather than fluctuations of the operator within given basis states? We do not expect that, even in such a basis, $\mathrm{q}_{5}, \mathrm{q}_{1}$, and $\mathrm{q}_{W}$ will be parametrically suppressed near $r=0$, but in such a basis, the fuzzball microstates would be reliable as possible.

\section{PROBING MULTICHARGE SOLUTIONS}

In the previous section, we provided a detailed discussion of the two-charge fuzzballs. However, the corresponding conventional solution has vanishing horizon area. So, it is of interest to investigate fuzzballs that have the same charges as black holes with a macroscopic horizon.
In this section, we will consider the class of asymptotically AdS fuzzball solutions discovered in Ref. [10] following previous work in Ref. [49]. The conventional black-hole geometry corresponding to these charges is given in Ref. [11]. We cannot repeat the analysis of Sec. III and compute quantum expectations and fluctuations since (a) all fuzzball solutions with the charges of Ref. [10] have not been discovered and (b) these solutions have not been quantized. Moreover, even in the set of solutions of Ref. [10], we will consider only a subset for which the form of the metric was explicitly given in Ref. [10].

These solutions are macroscopically distinguishable from the conventional black hole. Therefore, if we believe Expectation 2, then Result 2 immediately tells us that they cannot be typical elements of a basis. The calculations in this section show that one can reach this conclusion even without assuming Expectation 2 and simply by considering asymptotic observables.

The solutions of Ref. [10] are asymptotically AdS, and the asymptotic observable we will focus on is a two-point function of a marginal scalar operator in the boundary CFT. We will use this two-point function to investigate the energy gap between successive excitations of the fuzzball solutions. We find that this gap is too large and violates Expectation 1. We will also investigate whether fuzzball solutions satisfy a specific bound for the falloff of the twopoint function for large spacelike momenta. This bound holds in any conformal field theory and is saturated by the black-hole geometry. However, fuzzballs fail to saturate this bound, indicating that they cannot be typical microstates.

\section{A. Review of the solution}

The metric given in Ref. [10] is

$$
\begin{aligned}
d s_{6}^{2} & =-\frac{2}{\sqrt{\mathcal{P}}}(d v+\beta)\left(d u+\omega+\frac{1}{2} \mathcal{F}(d v+\beta)\right)+\sqrt{\mathcal{P}} d s_{4}^{2} ; \\
u & =(t-y) / \sqrt{2} ; \quad v=(t+y) / \sqrt{2} ; \quad y \sim y+2 \pi R_{y} ; \\
d s_{4}^{2} & =\frac{\Sigma d r^{2}}{r^{2}+a^{2}}+\Sigma d \theta^{2}+\left(r^{2}+a^{2}\right) \sin ^{2} \theta d \phi^{2}+r^{2} \cos ^{2} \theta d \psi^{2} ; \\
\mathcal{P} & =Z_{1} Z_{2}-Z_{4}^{2} ; \quad \beta=\frac{a^{2} R_{y}}{\sqrt{2} \Sigma}\left(\sin ^{2} \theta d \phi-\cos ^{2} \theta d \psi\right) ; \quad \Sigma=\left(r^{2}+a^{2} \cos ^{2} \theta\right) .
\end{aligned}
$$

The functions $Z_{1}, Z_{4}$, and $\mathcal{F}$ in the metric above depend on three integer parameters $k, m$, and $n$. In this paper, we will consider the simplest case, $k=1, m=0, n$ arbitrary, for which it is easy to write down explicit expressions for these quantities. For this case, we find that

$$
\begin{aligned}
& Z_{1}=\frac{\left(a^{2}+r^{2}\right)^{-n-1}\left(a^{2} b^{2} R_{y}{ }^{2} \sin ^{2}(\theta) r^{2 n} \cos \left(2\left(\frac{n(t+y)}{R_{y}}+\phi\right)\right)+2 Q_{1} Q_{5}\left(a^{2}+r^{2}\right)^{n+1}\right)}{2 Q_{5}\left(a^{2} \cos ^{2}(\theta)+r^{2}\right)} ; \\
& Z_{4}=\frac{a b R_{y} \sin (\theta) r^{n}\left(a^{2}+r^{2}\right)^{-\frac{n}{2}-\frac{1}{2}} \cos \left(\frac{n(t+y)}{R_{y}}+\phi\right)}{a^{2} \cos ^{2}(\theta)+r^{2}} ; \\
& \mathcal{F}=-\frac{1-r^{2 n}\left(a^{2}+r^{2}\right)^{-n}}{a^{2}}
\end{aligned}
$$


The parameters in the solution are related through the constraint

$$
\frac{Q_{1} Q_{5}}{R_{y}^{2}}=a^{2}+b^{2} / 2
$$

The asymptotic geometry of these solutions is $\mathrm{AdS}_{3} \times S^{3}$ with an AdS radius $\lambda=\left(Q_{1} Q_{5}\right)^{\frac{1}{4}}$. By the standard formula for the AdS central charge, we also have

$$
\frac{3 \lambda}{2 G_{3}}=6 N_{1} N_{5},
$$

where $N_{1}$ and $N_{5}$ are the numbers of D1 and D5 branes and $G_{3}$ is the three-dimensional Newton's constant. In addition, we set $\mathcal{N}=N_{1} N_{5} /\left(a^{2}+b^{2} / 2\right)$.

The charges of the solutions - the angular momenta along the $S^{3}\left(J_{L}, J_{R}\right)$, the mass $(M)$, and the momentum along the $y$ direction $\left(P_{y}\right)$ are given by

$$
J_{L}=J_{R}=\frac{\mathcal{N}}{2} a^{2} ; \quad M=P_{y}=\frac{\mathcal{N} n}{2 R_{y}} b^{2} .
$$

\section{B. Physical quantities of interest}

Let us consider a marginal scalar operator in the boundary theory. We will consider the Fourier transform of its Wightman function in a state dual to the fuzzball solution

$$
G(\omega, \gamma)=\int\langle f|O(t, y) O(0,0)| f\rangle e^{\frac{i \omega t}{R_{y}}} e^{\frac{i \gamma y y}{R_{y}}} d t d y
$$

It will also be useful to consider the Fourier transform of the commutator, which is just the difference of two Wightman functions

$$
\begin{aligned}
J(\omega, \gamma) & =\int\langle f|[O(t, y), O(0,0)]| f\rangle e^{\frac{i \omega t}{R_{y}}} e^{\frac{i \gamma y}{R_{y}}} d t d y \\
& =G(\omega, \gamma)-G(-\omega,-\gamma) .
\end{aligned}
$$

This Wightman function can be computed using the standard AdS/CFT dictionary by considering the boundary limit of a bulk minimally coupled massless scalar, $\phi$, with no motion along the $S^{3}$ coordinates,

$$
G(\omega, \gamma)=\lim _{r \rightarrow \infty} r^{4} \int\langle f|\phi(r, t, y) \phi(r, 0,0)| f\rangle e^{\frac{i \omega t}{R_{y}}} e^{\frac{i \gamma y}{R_{y}}} d t d y .
$$

This follows from the standard "extrapolate" dictionary in $\mathrm{AdS} / \mathrm{CFT}^{4}$

\footnotetext{
${ }^{4}$ In some special cases in $d=4$ that correspond to Coulomb branch solutions of $\mathcal{N}=4$ super yang mills, there are subtleties with the standard extrapolate dictionary [50], but these subtleties are irrelevant here.
}

It was shown in Refs. [16,51] that, in the fuzzball background under consideration, the massless scalar equation is separable. Furthermore, $t$ and $y$ are Killing vectors for the metric, and therefore it is convenient to expand the bulk scalar field as

$$
\phi(r, t, y)=\sum_{\omega, \gamma} a_{\omega, \gamma} R_{\omega, \gamma}(r) e^{\frac{-i \omega t}{R_{y}}} e^{\frac{-i \gamma y}{R_{y}}}+\text { H.c. }
$$

where the bulk operators are normalized so that $\left[a_{\omega, \gamma}, a_{\omega^{\prime}, \gamma^{\prime}}^{\dagger}\right]=\delta_{\omega \omega^{\prime}} \delta_{\gamma \gamma^{\prime}}$. We take the radial wave functions corresponding to different $\omega, \gamma$ to be orthonormal in the Klein-Gordon norm. Therefore, they satisfy

$$
\int h(r) \omega R_{\omega, \gamma}(r) R_{\omega, \gamma}^{*}(r) d r=1
$$

where the measure factor, $h(r)=8 \pi^{3} \sqrt{-g} /(\sin \theta \cos \theta)$, depends only on $r$. Note that this requires us to consider only normalizable radial bulk solutions.

The boundary Wightman function is then just given by

$$
G(\omega, \gamma)=N_{\omega, \gamma}\left|C_{\omega, \gamma}\right|^{2},
$$

where

$$
C_{\omega, \gamma}=\lim _{r \rightarrow \infty} r^{2} R_{\omega, \gamma}(r)
$$

and

$$
\left\langle f\left|a_{\omega, \gamma} a_{\omega^{\prime}, \gamma^{\prime}}^{\dagger}\right| f\right\rangle=N_{\omega, \gamma} \delta_{\omega, \omega^{\prime}} \delta_{\gamma \gamma^{\prime}}
$$

If the fuzzball state is approximately thermal, we expect that $N_{\omega, \gamma}=\frac{1}{1-e^{-\beta \omega}}$ and is independent of $\gamma$. However, we can avoid any assumptions about the function $N_{\omega, \gamma}$ by considering the commutator. Then, by using the fact that

$$
\left\langle f\left|\left[a_{\omega, \gamma}, a_{\omega^{\prime}, \gamma^{\prime}}^{\dagger}\right]\right| f\right\rangle=\delta_{\omega, \omega^{\prime}} \delta_{\gamma \gamma^{\prime}},
$$

we see that the commutator is simply given by

$$
J(\omega, \gamma)=\left|C_{\omega, \gamma}\right|^{2} .
$$

Therefore, the computation of the Wightman function and the commutator reduces essentially to a computation of $C_{\omega, \gamma}$, which can be obtained by solving the bulk radial equation and normalizing it under (4.4).

We will be particularly interested in the behavior of these functions in the limit in which $\gamma \gg 1$. In this limit, we can perform analytic calculations using a Wentzel-KramersBrillouin (WKB) approximation. We will show that $G(\omega, \gamma)$ and $J(\omega, \gamma)$ have support on a discrete set of frequencies with a gap between successive excitations that 
scales with $\frac{a^{2}}{b^{2}}$. Therefore, even if we take $\frac{a}{b} \ll 1$ (but not suppressed by an exponent of $N_{1} N_{5}$ ), we see by Expectation 1 that these states are very atypical.

The large- $\gamma$ limit of the Wightman function and the commutator is also of interest because, by virtue of having a horizon, black holes saturate a bound on how slowly these functions can decay at large $\gamma$. We will show, again, that the fuzzball solutions do not saturate this bound in the limit in which $\frac{a^{2}}{b^{2} n}=\mathrm{O}(1)$. Therefore, these fuzzball solutions also do not obey the ETH.

\section{Propagation of a massless scalar}

When the angular momentum of the mode along $S^{3}$ is zero, the wave equation, $\square \phi=0$, yields the following equation for the radial mode:

$$
\begin{aligned}
R_{\omega, \gamma}^{\prime \prime}(r) & +Q(r) R_{\omega, \gamma}^{\prime}(r)+P(r) R_{\omega, \gamma}(r)=0 ; \\
Q(r)= & \frac{a^{2}+3 r^{2}}{a^{2} r+r^{3}} ; \\
P(r)= & \frac{1}{4 a^{2} r^{2}\left(a^{2}+r^{2}\right)^{n+2}}\left(-b^{4} r^{2}(\omega-\gamma)^{2}\left(r^{2 n}-\left(a^{2}+r^{2}\right)^{n}\right)-4 a^{6} \gamma^{2}\left(a^{2}+r^{2}\right)^{n}\right. \\
& \left.-2 a^{2} r^{2}\left(b^{2}(\omega-\gamma)\left(r^{2 n}(\omega-\gamma)-2 \omega\left(a^{2}+r^{2}\right)^{n}\right)\right)+4 a^{4} r^{2}\left(a^{2}+r^{2}\right)^{n}\left(\omega^{2}-\gamma^{2}\right)\right) .
\end{aligned}
$$

With the appropriate translation of notation, this is the same as the wave equation derived in Ref. [16]. To put the equation in WKB form, we redefine

$$
R_{\omega, \gamma}(r)=\alpha \frac{\psi(r)}{\sqrt{r\left(r^{2}+a^{2}\right)}} .
$$

Here, $\alpha$ is a normalization constant that we will turn to in Sec. IV E, and we have suppressed the $\omega, \gamma$ dependence on $\psi$ and $\alpha$. Further, changing variables to $\xi=\frac{r}{a}$ and setting $b=a \kappa$, we can put the equation for $\psi$ in WKB form,

$$
\frac{d^{2} \psi(\xi)}{d \xi^{2}}-V(\xi) \psi(\xi)=0,
$$

with

$$
\begin{aligned}
V(\xi)= & \frac{1}{4\left(\xi^{2}+1\right)^{2}}\left[\frac{4 \gamma^{2}-1}{\xi^{2}}+4 \gamma^{2}+3 \xi^{2}+\kappa^{2}\left(\kappa^{2}+2\right)\right. \\
& \left.\times(\omega-\gamma)^{2} \frac{\xi^{2 n}}{\left(\xi^{2}+1\right)^{n}}-\left(\kappa^{2}(\omega-\gamma)+2 \omega\right)^{2}+6\right] .
\end{aligned}
$$

The potential has two turning points, and we can understand its qualitative behavior as follows. We see that at small $\xi(r \ll a)$ the potential is positive since it is dominated by the $\frac{4 \gamma^{2}-1}{\xi^{2}}$ term in the numerator of (4.8). We remind the reader that $\gamma \gg \omega$, and we will primarily be interested in a regime in which $\kappa \gg 1$. Then, it is clear that for a range of values of $\xi$ near $\xi=1$ the potential becomes negative before becoming positive again for large $\xi$ due to the $3 \xi^{2}$ term in the numerator. A graph of the potential is shown in Fig. 7 for some typical values of $\kappa, n$, and $\gamma$ with $\omega=0$ for simplicity.

For large values of $\gamma$, the WKB approximation is valid everywhere except very close to the two turning points or when $\xi=\mathrm{O}(\gamma)$. In this large $\xi$ region, we will match the WKB solution to a Bessel function, and we will deal with the turning points by interpolating between the two sides using the Airy functions.

Let us denote the position of the first turning point by $\xi_{1}$ and the second turning point by $\xi_{2}$. Then, for small $\xi$, if we insist that the solution be normalizable, we can write

$$
\psi(\xi)=\frac{1}{V(\xi)^{\frac{1}{4}}} e^{\int_{\xi_{1}}^{\xi} \sqrt{V(\zeta)} d \zeta}, \quad \xi<\xi_{1} .
$$

Near $\xi=\xi_{1}$, we can approximate $V(\xi) \approx\left|V^{\prime}\left(\xi_{1}\right)\right|\left(\xi_{1}-\xi\right)$, and therefore we have

$$
\psi(\xi)=\frac{2 \sqrt{\pi}}{\left|V^{\prime}\left(\xi_{1}\right)\right|^{\frac{1}{6}}} A i\left(\left|V^{\prime}\left(\xi_{1}\right)\right|\left(\xi_{1}-\xi\right)\right), \xi \approx \xi_{1},
$$

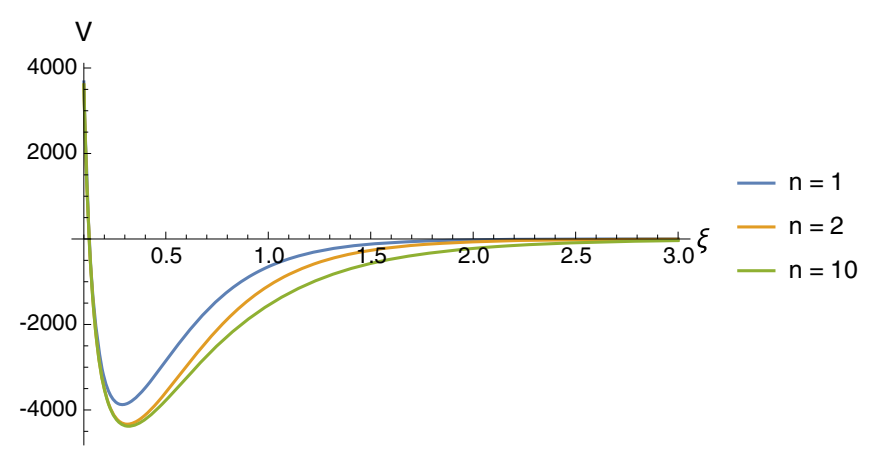

FIG. 7. A graph of $V(\xi)$ vs $\xi$ with $\gamma=10, \omega=0, \kappa=4$, and different values of $n$. 
where we have chosen the Airy Ai function based on the expected asymptotics for $\xi<\xi_{1}$. Matching again with the WKB solution, we find that

$$
\begin{aligned}
\psi(\xi) & =\frac{1}{|V(\xi)|^{\frac{1}{4}}}\left[e^{i \frac{\pi}{4}} e^{-i \int_{\xi_{1}}^{\xi} \sqrt{|V(\zeta)|} d \zeta}+e^{-i \frac{\pi}{4}} e^{i \int_{\xi_{1}}^{\xi} \sqrt{\mid V(\zeta)} d \zeta}\right] \\
& =\frac{1}{|V(\xi)|^{\frac{1}{4}}}\left[A_{-} e^{\frac{i \pi}{4}} e^{i \int_{\xi_{2}}^{\xi} \sqrt{|V(\zeta)|} d \zeta}+e^{-\frac{i \pi}{4}} A_{+} e^{-i \int_{\xi_{2}}^{\xi} \sqrt{|V(\zeta)|} d \zeta}\right] \\
\xi_{1} & <\xi<\xi_{2},
\end{aligned}
$$

where

$$
A_{ \pm}=e^{ \pm i \int_{\xi_{1}}^{\xi_{2}} \sqrt{|V(\zeta)|} d \zeta}
$$

Near $\xi \approx \xi_{2}$, we need to use the Airy function interpolation again and find that the solution matches to

$$
\begin{aligned}
\psi(\xi) & =\frac{1}{\mid V(\xi)^{\frac{1}{4}}}\left[B_{+} e^{\int_{\xi_{2}}^{\xi} \sqrt{|V(\zeta)|} d \zeta}+B_{-} e^{-\int_{\xi_{2}}^{\xi} \sqrt{|V(\zeta)|} d \zeta}\right], \\
\xi_{2} & <\xi \ll \gamma,
\end{aligned}
$$

with $B_{+}=\frac{A_{-}+A_{+}}{2} ; \quad B_{-}=\frac{i}{2}\left(A_{-}-A_{+}\right)$. Now, for very large $\xi$, we can also solve for the wave equation in terms of Bessel functions

$$
\psi(\xi)=\frac{2 C}{\delta} \sqrt{\xi} I_{1}\left(\frac{\delta}{\xi}\right), \quad \xi \gg 1,
$$

where $\delta^{2}=\frac{1}{2}\left(\gamma^{2}-\omega^{2}\right)\left(2+\kappa^{2}\right)$, and we have picked the Bessel I function by demanding that the solution be normalizable at infinity. Matching the solutions (4.12) and (4.13) in the neighborhood of some point $\xi_{3}$ which satisfies $1 \ll \xi_{3} \ll \gamma$, where both solutions are valid, we see that the solutions can only match if $B_{+}=0$. This simply tells us that in the region where the potential becomes positive again the magnitude of the wave function cannot grow exponentially.

This gives us a quantization condition on the potential,

$$
2 \int_{\xi_{1}}^{\xi_{2}}|V(\zeta)|^{\frac{1}{2}} d \zeta=(2 m+1) \pi
$$

for some integer $m$. From the formula above, we have $B_{-}=(-1)^{m}$, and this additionally tells us that

$$
\begin{aligned}
\frac{2 C}{\sqrt{2 \pi}} \frac{\xi_{3}}{\delta^{\frac{3}{2}}} e^{\frac{\delta}{\xi_{3}}} & =\frac{(-1)^{m}}{V\left(\xi_{3}\right)^{\frac{1}{4}}} e^{-\int_{\xi_{2}}^{\xi_{3}}|V(\zeta)|^{\frac{1}{2}} d \zeta} \\
& \Rightarrow C=(-1)^{m} \frac{\delta}{2} \sqrt{2 \pi} e^{-\int_{\xi_{2}}^{\xi_{3}} \mid V(\zeta)^{\frac{1}{2}} d \zeta-\frac{\delta}{\xi_{3}}}
\end{aligned}
$$

It is clear that the value of $C$ does not depend on the precise value of $\xi_{3}$ chosen to perform the matching.

We will now use this WKB solution to compute some physical quantities of interest.

\section{Energy gap}

First, we consider the gap in energies of states in the neighborhood of the fuzzball state. This is a question of the values of $\omega$ for which $G(\omega, \gamma)$ has support.

Clearly, $G(\omega, \gamma)$ vanishes for those frequencies in which no normalizable solution exists. (This is independent of any assumption about $N_{\omega, \gamma}$.) So, the energy gap can be obtained by examining the values of $\omega$ for which the quantization condition (4.14) is satisfied. The quantization condition can be parsed as follows. First, in the limit $\xi_{1}<\xi<\xi_{2}$, we expand the square root of the potential as

$$
\begin{aligned}
\sqrt{|V|} & =V_{\frac{1}{2}, 1} \gamma-\omega \kappa^{2} V_{\frac{1}{2}, 0}+\mathrm{O}\left(\frac{1}{\gamma}\right), \\
V_{\frac{1}{2}, 1} & =\frac{\sqrt{\left|\kappa^{4}\left(\left(\frac{1}{\xi^{2}}+1\right)^{n}-1\right)-2 \kappa^{2}-4\left(\frac{1}{\xi^{2}}+1\right)^{n+1}\right|}}{2 \xi^{2}\left(\frac{1}{\xi^{2}}+1\right)^{\frac{n}{2}+1}}, \\
V_{\frac{1}{2}, 0} & =\frac{\left(\kappa^{2}+2\right)\left(\left(\frac{1}{\xi^{2}}+1\right)^{n}-1\right)\left(\frac{1}{\xi^{2}}+1\right)^{-\frac{n}{2}-1}}{2 \xi^{2} \sqrt{\left|\kappa^{4}\left(\left(\frac{1}{\xi^{2}}+1\right)^{n}-1\right)-2 \kappa^{2}-4\left(\frac{1}{\xi^{2}}+1\right)^{n+1}\right|}} .
\end{aligned}
$$

The absolute value sign inside the square root is for later use and does not have any effect in the range under consideration.

Now, at large $\gamma$, the values of $\xi_{1}$ and $\xi_{2}$ are controlled by $V_{\frac{1}{2}, 1}$ and do not depend on $\omega$. Therefore, if we consider two consecutive solutions of (4.14) that differ by an amount $\delta \omega$, then this difference must satisfy

$$
\delta \omega=\frac{\pi}{\kappa^{2} g_{n}},
$$

where

$$
g_{n}=\int_{\xi_{1}}^{\xi_{2}} V_{\frac{1}{2}, 0} d \xi
$$

For general values of $\kappa$, this condition can only be solved numerically, but it is of interest to examine the limit in which $\kappa \gg 1$. In this limit, we can approximate $\xi_{1} \approx \frac{2}{\kappa^{2}}$ and $\xi_{2} \approx \kappa \sqrt{\frac{n}{2}}$. For the calculation of $g_{n}$, these limits are effectively 0 and $\infty$. Therefore, expanding $V_{\frac{1}{2}, 0}$ at large $\kappa$, we find 


$$
g_{n}=\int_{0}^{\infty} d \xi \frac{\sqrt{\left(\frac{1}{\xi^{2}}+1\right)^{n}-1}\left(\frac{1}{\xi^{2}}+1\right)^{-n / 2}}{2\left(\xi^{2}+1\right)}+\mathrm{O}\left(\frac{1}{\kappa}\right) .
$$

The first few values of $g_{n}$ (for $n=1$ to $n=5$ ) are $\{0.5,0.574,0.610,0.632,0.648\}$.

Therefore, at large $\gamma$ and large $\kappa$, the energy gap between consecutive excitations scales as $\frac{1}{\kappa^{2}}$ with a simple numerical prefactor.

\section{E. Large- $\gamma$ Wightman function and commutator}

We now briefly explain the significance of the behavior of the Wightman function and the commutator at large $\gamma$ but small $\omega$. As explained in Ref. [29], in any conformal field theory, the large- $\gamma$, small- $\omega$, limit of the thermal Wightman function/commutator must fall off exponentially, with an exponent that is bounded below. We review this argument below. In Ref. [29], it was also shown that black holes saturate this bound because of the presence of the horizon. It is therefore of interest to understand whether fuzzballs also saturate this bound.

We will perform the analysis for the Wightman function below, although the analysis for the commutator is precisely the same. To obtain the bound on the behavior of the Wightman function, we consider this correlator in a state with a finite temperature and chemical potential for the momentum in the $y$ direction in some arbitrary two-dimensional conformal field theory living on a circle with radius $R_{y}$,

$$
\begin{aligned}
G(t, y)= & \operatorname{Tr}\left(e^{-\beta\left(H-\mu P_{y}\right)} O(t, y) O(0,0)\right) \\
= & \sum_{m, n} e^{-\beta\left(E_{m}-\mu P_{m}\right)} \\
& \times e^{-i t\left(E_{n}-E_{m}\right)-i y\left(P_{n}-P_{m}\right)}|\langle m|O(0,0)| n\rangle|^{2},
\end{aligned}
$$

where the sum over $m, n$ runs over a complete set of energy/ momentum eigenstates and to lighten the notation we have the same symbol for the position-space Wightman function as for its Fourier transform. Now, by Fourier transforming in time, we find that

$$
\begin{aligned}
G(\omega, y)= & \int G(t, y) e^{\frac{i \omega t}{R_{y}}} d t \\
= & 2 \pi \sum_{m, n} \delta\left(E_{n}-\frac{\omega}{R_{y}}-E_{m}\right) \\
& \times e^{-\beta\left(E_{m}-\mu P_{m}\right)-i\left(P_{m}-P_{n}\right) y}|\langle m|O(0,0)| n\rangle|^{2} .
\end{aligned}
$$

Now, writing $y=y_{r}+i y_{i}$, and using the spectrum condition $E_{m} \geq\left|P_{m}\right|$, we see that the real part of the exponent in the sum over $m, n$ can be written as

$$
\begin{aligned}
& \operatorname{Re}\left(-\beta\left(E_{m}-\mu P_{m}\right)-i\left(P_{m}-P_{n}\right) y\right) \leq-\beta E_{m}(1-|\mu|)+\left(\left|P_{m}\right|+\left|P_{n}\right|\right)\left|y_{i}\right| \\
& =\beta(1-|\mu|) \frac{\omega}{2 R_{y}}-\left(E_{m}+E_{n}\right) \frac{\beta(1-|\mu|)}{2}+\left(\left|P_{m}\right|+\left|P_{n}\right|\right)\left|y_{i}\right| \\
& \leq \beta(1-|\mu|) \frac{\omega}{2 R_{y}}-\left(E_{m}+E_{n}\right)\left(\frac{\beta(1-|\mu|)}{2}-\left|y_{i}\right|\right) .
\end{aligned}
$$

Therefore, the exponent always supplies a convergence factor in the sum over $m, n$, provided that $|\operatorname{Im}(y)|<\frac{\beta(1-|\mu|)}{2}$, and therefore the Green function can be analytically continued in the $y$ plane in both directions up to this limit. But then writing

$$
G\left(\omega, y_{r}-i y_{i}\right)=\int_{\gamma=-\infty}^{\infty} G(\omega, \gamma) e^{\frac{-i \gamma\left(y_{r}+y_{i}\right)}{R_{y}}},
$$

we see that, in the regime in which $\gamma \rightarrow \infty$, this is only possible if

$$
\lim _{\gamma \rightarrow \infty} \frac{-\log |G(\omega, \gamma)|}{\left(|\gamma| / R_{y}\right)} \geq \frac{\beta(1-|\mu|)}{2} .
$$

Note that the minus sign outside the log indicates that the Wightman function must decay at large $\gamma$. Second, we also note that this bound can be written in terms of the left and right temperatures that couple to the left and right Virasoro charges: $\beta_{L}=\frac{1}{R_{y}} \beta(1-\mu) ; \beta_{R}=\frac{1}{R_{y}} \beta(1+\mu)$, if we recognize that $\beta(1-|\mu|)=R_{y} \min \left(\beta_{L}, \beta_{R}\right)$. In this notation, the bound simply becomes

$$
\lim _{\gamma \rightarrow \infty} \frac{-\log |G(\omega, \gamma)|}{\gamma} \geq \frac{1}{2} \min \left(\beta_{L}, \beta_{R}\right) .
$$

Repeating the analysis above, we see that the same bound also applies to the commutator (4.3)

$$
\lim _{\gamma \rightarrow \infty} \frac{-\log |J(\omega, \gamma)|}{\gamma} \geq \frac{1}{2} \min \left(\beta_{L}, \beta_{R}\right) .
$$

It was explained in Ref. [29] that black holes saturate this bound. Intuitively, this happens for the following reason. 
In general, modes with large $\gamma$ but small $\omega$ are unusual because they have larger momentum than frequency and are "spacelike" near the boundary. However, the black-hole horizon allows such modes to propagate in the bulk because of the redshift near the horizon. The fuzzball also has a redshift, but we will see below that fuzzballs do not saturate (4.17).

To calculate the large- $\gamma$ behavior of the Wightman function and the commutator, we need to compute $\alpha$ defined in (4.6) and the asymptotic behavior of $\psi$. We can compute

$$
\alpha^{-2}=\int_{0}^{\infty} \omega \frac{|\psi(r)|^{2}}{r\left(r^{2}+a^{2}\right)} h(r) d r
$$

where the measure factor, $h(r)$, is given below (4.4).

However, we see that the WKB wave function given in (4.9), (4.11), and (4.13) has no growing exponential of $\gamma$ and therefore

$$
\lim _{\gamma \rightarrow \infty} \frac{\log (\alpha)}{\gamma}=0 .
$$

This leaves us with the asymptotic part of the wave function, which is controlled by the coefficient $C$ in (4.15). A simple calculation yields that at large $\gamma$ we have

$$
\begin{aligned}
-\log (C) & =\int_{\xi_{2}}^{\xi_{3}} \sqrt{V(\xi)} d \xi+\frac{\delta}{\xi_{3}}+\mathrm{O}(1) \\
& =\frac{\pi \gamma\left(\left(8 \kappa^{2}+11\right) n-1\right)}{32 \kappa^{2} n^{3 / 2}}+\mathrm{O}(1) .
\end{aligned}
$$

Using the formula (4.5), the falloff of the Wightman function and the commutator for the fuzzball geometry is given by

$$
\begin{aligned}
\lambda_{\text {fuzz }} & \equiv \lim _{\gamma \rightarrow \infty} \frac{\log |G(\omega, \gamma)|}{\gamma}=\lim _{\gamma \rightarrow \infty} \frac{\log |J(\omega, \gamma)|}{\gamma} \\
& =\frac{\pi}{2 \sqrt{n}}+\frac{(11 n-1) \pi}{16 n^{\frac{3}{2}} \kappa^{2}} .
\end{aligned}
$$

One subtlety in comparing the fuzzball result with the bound is that the fuzzballs also have angular momentum along the $S^{3}$. It is understood holographically that-at least for the purpose of computing correlation functions such as (4.2), which do not depend on any $S^{3}$ variable themselves-black holes with angular momentum along the $S^{3}$ direction behave as if they have an "effective" virasoro charge given by

$$
L_{0}^{\mathrm{eff}}=L_{0}-\frac{J_{L}^{2}}{N_{1} N_{5}}
$$

where we have recalled that the central charge of the theory is $6 N_{1} N_{5}$. (See, e.g., the discussion below (5.17) of
Ref. [52] and the original discussion in Ref. [53].) We emphasize that using this "effective charge" rather than the original charge only weakens the bound (4.17) and so makes the comparison more favorable for fuzzballs.

The right inverse temperature of the fuzzball is infinity because the solution satisfies $\bar{L}_{0}=0$. The effective left inverse temperature corresponding to the effective charge above is given by

$$
\beta_{L}=\pi\left(\kappa^{2}+2\right) \sqrt{\frac{1}{\kappa^{2}\left(\kappa^{2}+2\right) n-1}} .
$$

Comparing the decay of the fuzzball Wightman function and the commutator to the bound, we find that

$$
\lambda_{\text {fuzz }}-\frac{1}{2} \beta_{L}=\frac{\pi(3 n-1)}{16 \kappa^{2} n^{3 / 2}}+\mathrm{O}\left(\frac{1}{\kappa^{4}}\right) .
$$

Therefore, fuzzballs fail to saturate the large- $\gamma$ bound (4.17) by the amount shown above.

\section{F. Numerical verification}

We can verify the analytic results above by direct numerical analysis of the propagation of a scalar field in the fuzzball background.

We consider a fuzzball background with a given value of $\kappa$ and $n$ and a scalar field excitation with a given value of $y$ momentum, $\gamma$. The Eq. (4.7) is subject to normalizability under the Klein-Gordon norm, and this fixes boundary conditions at both $\xi=0$ and $\xi=\infty$. In fact, both $\xi=0$ and $\xi=\infty \mathrm{s}$ are singular points of the equation, and to solve the equation numerically, we must expand in a series solution about the point $\xi=0$ out to $\xi=\epsilon$.

Near $\xi=0$, we set the function and its derivative through the expansion

$$
\psi^{0}(\xi)=\left(\frac{\xi}{\epsilon}\right)^{\frac{2 \gamma-1}{2}}\left(1+a_{0} \xi^{2}\right), \quad 0<\xi \leq \epsilon,
$$

with

$$
a_{0}=-\frac{\gamma^{2}\left(\kappa^{4}+4\right)+\left(\kappa^{2}+2\right)^{2} w^{2}-2 \gamma \kappa^{2}\left(\kappa^{2}+2\right) w-8}{16(\gamma+1)} .
$$

Note that, for numerical convenience, the normalization used here is different from the normalization used in (4.9).

Near $\xi=\infty$, we set the function and its derivative through the expansion

$$
\psi^{\infty}=\frac{1}{\sqrt{\xi}}\left(1+\frac{a_{\infty}}{\xi^{2}}\right), \quad \frac{1}{\epsilon} \leq \xi<\infty,
$$

with 


$$
\begin{aligned}
a_{\infty}= & \frac{1}{32}\left(-\gamma^{2} \kappa^{4}+4 \gamma^{2}-\omega^{2} \kappa^{4}-4 \kappa^{2} \omega^{2}\right. \\
& \left.-4 \omega^{2}+2 \gamma \kappa^{4} \omega+4 \gamma \kappa^{2} \omega\right) .
\end{aligned}
$$

Note that, for numerical convenience, this normalization is also chosen to be different from the normalization used in (4.13).

The allowed values of $\omega$ can then be fixed by a shooting procedure. Given a guess for $\omega$, starting from $\xi=\epsilon$, we solve the equation to the midpoint of the trough of the potential: $\xi_{\mathrm{m}}=\frac{1}{2}\left(\xi_{1}+\xi_{2}\right)$. This solution yields some values for the function and its derivative: $\psi^{0}\left(\xi_{\mathrm{m}}\right)$ and $\frac{d \psi^{0}\left(\xi_{\mathrm{m}}\right)}{d \xi}$. Similarly, for the same value of $\omega$, we can start from $\xi=\frac{1}{\epsilon}$ and solve inward to obtain a second set of values for the function and its derivative: $\psi^{\infty}$ and $\frac{d \psi^{\infty}}{d \xi}$. These values define a difference function for any given value of $\omega$,

$$
\mathcal{D}(\omega)=\frac{d \psi^{\infty}}{d \xi} \psi^{0}\left(\xi_{\mathrm{m}}\right)-\frac{d \psi^{0}\left(\xi_{\mathrm{m}}\right)}{d \xi} \psi^{\infty}
$$

We then use nonlinear root-finding techniques to find the roots of $\mathcal{D}(\omega)$. In our analysis, we first bracketed the root and then used the Brent method as implemented in the GNU Scientific Library [54]. Bracketing methods are robust and guaranteed to converge to a root in the bracketed interval.

Note that it is because the equation is linear that we can get away with just matching the ratio of the function and its derivative at the point $\xi_{\mathrm{m}}$. If this were not the case, we would have to match both these quantities separately; this would force us to use two-dimensional root finding, which is far less robust.

The asymptotic value of the function, $C$, as defined above, can be fixed as follows. We denote the value that the solution starting at $\xi=0$ takes at $\xi_{1}$ by $\psi^{0}\left(\xi_{1}\right)$. Then, $C$ is given by

$$
C=\frac{\psi^{0}\left(\xi_{\mathrm{m}}\right)}{\psi^{\inf }\left(\xi_{\mathrm{m}}\right)} \times \frac{2 \sqrt{\pi} A i(0)}{\left|V^{\prime}\left(\xi_{1}\right)\right|^{\frac{1}{6}}} \times \frac{1}{\psi^{0}\left(\xi_{1}\right)} .
$$

Note that $A i(0) \approx 0.355028$. This formula implements the following procedure. First, we normalize the solution on the left so that it takes on the value given by (4.10) at $\xi_{1}$. Then, we normalize the solution on the right so that it matches the left solution at the midpoint.

Unlike the WKB analysis, the numerical analysis is not restricted to large $\gamma$. However, we can use it in the same regime to verify the results of the WKB approximation above.

In Fig. 8, we show how the gap between the first two nonzero solutions of $\omega$ matches with the analytic formula (4.16). We see that, at large $\kappa$ [which is the regime in which (4.16) is derived], the agreement is excellent. In Fig. 9, we show a comparison of the numerically computed asymptotic value for $C$ with the analytic formula (4.15) for

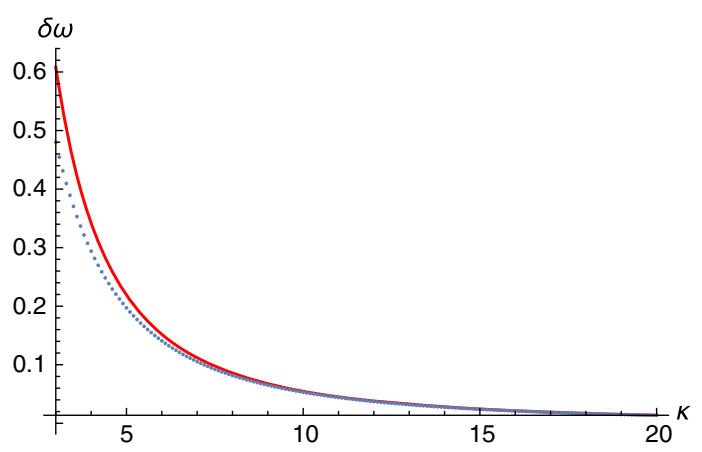

FIG. 8. Comparison between a numerical calculation (dots) of the gap between the first two allowed frequencies and the formula (4.16) (solid curve). Other parameters are $\gamma=100$, $n=2$. In its regime of validity (large $\kappa$ ), the formula (4.16) shows excellent agreement with the numerical results.

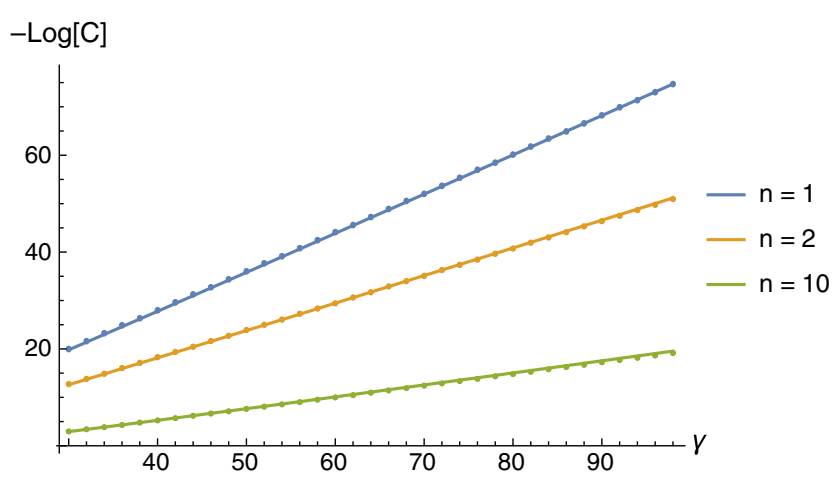

FIG. 9. Comparison between a numerical calculation (dots) of the asymptotic value $C$ with the formula (4.15) (solid curves) for different values of $n$ and $\gamma$. Here, $\kappa=5$ is held fixed. In the appropriate regime of validity of the analytic formula (large $\gamma$ ), it agrees very well with the numerical results.

a fixed value of $\kappa=5, n=2$ and varying values of $\gamma$. We see, once again, that the agreement is excellent in the regime in which the analytic formula is valid.

\section{G. Analysis of the result}

The key results derived above are the formula for the mass gap (4.16) and the decay of the Wightman function (4.18) at large $\gamma$, which we also verified numerically. We discuss their significance in turn.

The formula for the gap (4.16) tells us that the gap between the frequency of successive excitations of these solutions is far too large for a typical microstate. As described in the discussion around (2.2), Expectation (1) tells us that the Wightman function (after a small amount of local smearing) should have continuous support in frequency space. As explained there, this should be true even of the Wightman function in a BPS state. Even though the original state is BPS, the scalar excitation should connect these states to nearby non-BPS states that are expected to satisfy Expectation 1. 
However, for the fuzzball solutions, we find that the support is concentrated on discretely spaced frequencies with a gap between consecutive frequencies that scales with $\frac{1}{\kappa^{2}}$. This means that the fuzzball solutions that we have analyzed, with any finite value of $\kappa$ (provided $\kappa$ does not scale with the central charge), cannot serve as microstates of a black hole.

Instead, the finite energy gap is reminiscent of a phase of zero entropy-like thermal AdS as explained below (2.3). This suggests that the states corresponding to this set of fuzzball solutions belong to such a phase-which comprises exponentially atypical states.

The formula for the decay at large $\gamma$ (4.18) tells us that if fuzzballs are microstates then the set of fuzzball solutions corresponds to states that violate the ETH. This is because, on the basis of the black-hole calculation, the thermal state is expected to saturate the bound (4.17). The ETH would then suggest that eigenstates of the Hamiltonian or, indeed, typical elements of any other basis that spans that microcanonical ensemble should also saturate the bound.

We expect that the holographic theory dual to black holes should be chaotic in the supergravity regime, and therefore it should satisfy the ETH; the hypothesis that fuzzballs are black-hole microstates contradicts this expectation.

Second, note that, even if we disregard the ETH, the idea that fuzzballs are black-hole microstates leads to a strange conclusion: since the set of fuzzballs we have analyzed is below the bound, there must be some other fuzzballs that violate the bound (4.17). This is the only way that the microcanonical average can saturate the bound. Now, strictly speaking, this is not a contradiction since the bound (4.17) is a bound for the behavior of the thermal state in different theories and does not control the behavior of specific pure states. But, on the other hand, we are not aware of any geometry that violates the bound (4.17). In the absence of such an example, it seems difficult to understand how the fuzzball geometries could represent black-hole microstates.

\section{Very large values of $\kappa$}

We now briefly consider the limit in which we take $a \sim \ell_{\mathrm{pl}}$. In this limit, $\kappa$ becomes large, and the decay of the large- $\gamma$ Wightman function approaches the bound (4.17). The energy gap becomes small. ${ }^{5}$ However, this energy gap is still too large since it scales with an inverse power of the central charge rather than being exponentially suppressed. So, even in this limit, the fuzzball solutions do not yield the correct gap expected in the boundary theory. This, by itself, ensures that even Planck-sized fuzzballs do not have the right properties expected of a typical microstate.

We now discuss some independent problems with the idea of considering fuzzballs with Planckian features. We will argue that for $a \sim \ell_{\mathrm{pl}}$ such solutions become indistinguishable from the black hole in most of space, and quantum fluctuations are likely to be large in the near-horizon region where the fuzzball deviates from the black hole.

First, note that in this limit the angular momentum in the $S^{3}$ directions, which is proportional to $a^{2}$, vanishes. So, in the subsector under consideration, solutions with very long throats (small values of $a$ ) cannot correspond to states with arbitrary charges. However, for the remainder of this section, we will assume that when the full set of fuzzball geometries is found it will be possible to keep $a$ arbitrarily small, while keeping the charges constant by changing some other parameters.

Second, we note that the $a \rightarrow 0$ limit of (4.1) does not commute with the $r \rightarrow 0$ limit. If we first take $a \rightarrow 0$ so that we can neglect terms of order $\frac{a}{r}$, the metric has the following smooth limit:

$$
\begin{aligned}
d s_{6}^{2} \underset{a \rightarrow 0}{\rightarrow} \frac{\left(b^{2} n-2 r^{2}\right)}{\sqrt{2} b R_{y}} d t^{2}+\frac{\left(b^{2} n+2 r^{2}\right)}{\sqrt{2} b R_{y}} d y^{2}+\frac{b R_{y}}{\sqrt{2} r^{2}} d r^{2}+\frac{\sqrt{2} b n}{R_{y}} d t d y \\
+\frac{b R_{y} \cos ^{2}(\theta)}{\sqrt{2}} d \psi^{2}+\frac{b R_{y} \sin ^{2}(\theta)}{\sqrt{2}} d \phi^{2}+\frac{b R_{y}}{\sqrt{2}} d \theta^{2} .
\end{aligned}
$$

A change of variables to $\rho=\left(r^{2}+\frac{b^{2} n}{2}\right)^{\frac{1}{2}}$ shows that this is the metric of an extremal BTZ black hole $\times S^{3}$.

On the other hand, even when $\frac{b}{a} \gg 1$, if we explore the regions of the geometry where $r=\mathrm{O}(a)$, we find a different answer. In coordinates where $r=a \xi$, the metric expanded to $\mathrm{O}\left(\xi^{2}\right)$ near $\xi=0$ is given by

$$
\begin{aligned}
d s_{6}^{2}= & -\frac{\left(2 a^{2}-b^{2}\right)\left(\cos ^{2}(\theta)+\xi^{2}\right)}{2 \lambda^{2}} d t^{2}+\frac{b^{2}\left(\cos ^{2}(\theta)+\xi^{2}\right)}{\lambda^{2}} d t d y+\frac{\left(2 a^{2}+b^{2}\right)\left(\cos ^{2}(\theta)+\xi^{2}\right)}{2 \lambda^{2}} d y^{2} \\
& +\lambda^{2}\left(1-\xi^{2}\right) d \xi^{2}-2 \frac{a^{2} \sin ^{2}(\theta)}{\sqrt{a^{2}+\frac{b^{2}}{2}}} d t d \phi-\frac{b^{2} \cos ^{2}(\theta)}{\sqrt{a^{2}+\frac{b^{2}}{2}}} d t d \psi-2 \sqrt{a^{2}+\frac{b^{2}}{2}} \cos ^{2}(\theta) d y d \psi \\
& +\lambda^{2} d \theta^{2}+\lambda^{2} \sin ^{2} \theta d \phi^{2}+\lambda^{2} \cos ^{2} \theta d \psi^{2} .
\end{aligned}
$$

\footnotetext{
${ }^{5}$ This is the gap computed in Ref. [16] by taking $\kappa=N_{1} N_{5}$.
} 
If we take $a \sim \ell_{\mathrm{pl}}$, then the fuzzball metric has Planckian structures near $r=0$, and these structures are given by the metric in (4.19). Remarkably, curvature invariants such as the Ricci scalar and even the square of the Riemann tensor, $R_{\mu \nu \rho \sigma} R^{\mu \nu \rho \sigma}$, which can be computed from (4.19), remain finite in limit as $a \rightarrow 0$. So, in this limit, the fuzzball metric cleverly introduces Planckian structures, without introducing Planckian curvatures. This is a surprising and nice feature of the solution.

However, it would be incorrect to imagine that this makes the classical metric immune to quantum fluctuations in this region. The study of Sec. III tells us that we must also take into account quantum fluctuations in the parameters that specify a solution. In the two-charge case, the solution was specified by a profile function $F^{i}(s)$. But in the quantum theory, the profile function did not have a definite value because of the nonzero commutator, $\left[F^{i}(s), F^{i}(\tilde{s})\right]$. We saw that when the metric had Planckscale features the uncertainties in the profile function were enough to make these features unreliable.

In the absence of a moduli-space quantization of the solutions examined in this section, we cannot make analogous precise statements for the multicharge solutions. However, a rule of thumb is that we do not expect to pin down bulk length scales in a theory of quantum gravity with perfect certainty. Therefore, it is fair to estimate that in the quantum states corresponding to the metrics examined in this section the length scale $a$ will itself fluctuate and that $\delta a=\mathrm{O}\left(\ell_{\mathrm{pl}}\right)$.

If this is correct, then in the regime in which $a=\mathrm{O}\left(\ell_{\mathrm{pl}}\right)$, we also have $\frac{\delta a}{a}=\mathrm{O}(1)$. But then, examining the metric (4.19), we see that such fluctuations will induce fluctuations in the metric so that $\delta g_{\mu \nu}=\mathrm{O}\left(g_{\mu \nu}\right)$. For example, we see that for the determinant of the metric (4.19)

$$
g=\operatorname{det}\left(g_{\mu \nu}\right)=a^{4} \lambda^{4} \xi^{2}\left(\xi^{4}-1\right) \sin ^{2}(\theta) \cos ^{2}(\theta),
$$

and therefore

$$
\delta g=\frac{\partial g}{\partial a} \delta a=4 g \frac{\delta a}{a} .
$$

If $\frac{\delta a}{a}=\mathrm{O}(1)$, then $\frac{\delta g}{g}=\mathrm{O}(1)$. So, while the Planckian structures are smooth, in the sense that local curvature invariants remain bounded, they are nevertheless not reliable features of the metric.

We caution the reader that our arguments in this last paragraph have been necessarily somewhat imprecise. This is because the fuzzball program has itself not been carried through to completion in this setting. But we believe that our reasoning is robust for a simple reason. The parameters that specify a fuzzball solution are coordinates on the phase space of gravity. Usually, we do not consider classical solutions of which the distinctive features depend on specifying phase-space coordinates to an accuracy that depends on $\hbar$. Conversely, if we attempted to do so, we should expect that the minimal fluctuations on phase space induced by the uncertainty principle would wash out these features.

\section{CONCLUSIONS}

In this paper, we examined the fuzzball program by checking its consistency with some general results from statistical mechanics and some simple physical expectations. While the differences between typical black-hole microstates are exponentially suppressed, and so cannot be described geometrically, we showed that fuzzballs cannot even provide a reliable basis for the space of microstates.

We argued that fuzzballs that differ macroscopically from the conventional black hole are too atypical to be elements of the basis of microstates. We checked in Sec. IV that such macroscopic deviations can be detected by simple asymptotic correlators. We also showed that fuzzballs that cap off a macroscopic distance away from the horizon have a gap between the frequency of allowed excitations that is too large for black-hole microstates. We emphasize that, although our calculations were limited to specific sets of geometries, we expect this fact to generalize to arbitrary solutions that have macroscopic features.

These results imply that viable microstates must resemble the black hole very closely all the way up to Planck length from the horizon, where they must suddenly deviate from the black-hole geometry so that space ends before a horizon forms. However, we argued that in this regionjust where fuzzballs start to show interesting deviations from the black-hole geometry-the solutions are also expected to become unreliable since quantum fluctuations become of the same order as classical expectation values of components of the metric.

We verified this expectation through a detailed consideration of the two-charge fuzzball geometries. Here, using the well-known quantization of this set of solutions, we were able to compute the expectation values and the quantum fluctuations for the harmonic functions that enter the metric and for a 1-form that probes the geometry. At the end of these involved calculations, we found that, just as expected, typical microstates start to deviate from the conventional geometry only at the Planck scale, and quant um fluctuations make the solutions unreliable precisely in this region.

In the two-charge case, the conventional geometry has no horizon. However, whenever conventional black holes do have a regular horizon, the black-hole geometry continues to be reliable well past the horizon of the black hole and so- - unlike the geometry of fuzzballs - there is no reason to distrust the black-hole geometry at the horizon scale. So, our analysis supports the standard picture that the distinct microstates of a macroscopic black hole are all represented by the same geometry with a regular horizon. 
The fuzzball program is sometimes supported through indirect arguments-by suggesting that the information paradox necessitates the existence of structure at the horizon. We explained in Sec. II that these indirect arguments rely on an assumption of exact locality in quantum gravity. But extensive evidence from AdS/CFT and string theory suggests that this assumption is incorrect.

Our results are consistent with the results of Sen [40]. Sen pointed out that, in the context of the two-charge system, in the type II-B duality frame, the ground states of the D1-D5 system could not be represented by a stringscale black hole. This led Sen to suggest that fuzzballs should be understood as parametrizing the "hair" around a black hole, and not the microstates of the black hole itself. Where fuzzball solutions exist, Sen's argument suggests that quantizing the moduli space of fuzzballs should not be expected to reproduce the Bekenstein-Hawking entropy; rather, the entropy obtained by quantizing classical supergravity solutions should be added to the BekensteinHawking entropy to obtain the full degeneracy of the system.

Our results rely on some physical assumptions, two of which are outlined in Expectations 1 and 2. For the fuzzball program to be correct, some of these assumptions would have to be violated. For example, if it could be shown that, for some reason, even the Euclidean black hole is an inadmissible saddle point to compute thermal averages, then this could be used to invalidate our estimate of fluctuations in the metric in Eq. (2.7). This would open the door to allowing fuzzballs that can be described classically and perhaps differ from the conventional solution at the string scale.

At the same time, the fuzzball program would also have to explain why two-point functions of asymptotic operators computed in fuzzball microstates do not display a continuous spectrum. In AdS/CFT, this would suggest that either the boundary theory has a very exotic set of couplings-so that fuzzball microstates span the microcanonical ensemble but yet are not connected to most nearby energy eigenstates by the action of light primary operators - or exact degeneracies in energy eigenvalues are somehow restored at the supergravity point. These possibilities seem rather implausible.

It seems more plausible to us that fuzzballs should simply be thought of as "stars" with the same charges as black holes and not as microstates of the black hole. In this context, fuzzballs are very interesting solutions. The idea of stabilizing a self-gravitating system against gravitational collapse by causing space to end before a horizon is formed is also remarkably rich. The asymptotically AdS fuzzball solutions that have been found so far appear to represent valid states in the AdS/CFT correspondence, and it is an interesting problem to study their properties and determine the CFT duals to these states.

\section{ACKNOWLEDGMENTS}

We would like to thank Junggi Yoon for collaboration in the early stages of this work. We are grateful to Iosif Bena, Jan de Boer, Emil Martinec, Bidisha Chakrabarty, Monica Guica, Stefano Giusto, Dileep Jatkar, Jared Kaplan, Samir Mathur, Shiraz Minwalla, Yasunori Nomura, Kyriakos Papadodimas, Andrea Puhm, Ashoke Sen, Rodolfo Russo, Yogesh Srivastava, Kostas Skenderis, Bo Sundborg, Marika Taylor, Amitabh Virmani, and Nick Warner for helpful discussions. We are grateful to Iosif Bena and Masaki Shigemori for sharing their notes on the solutions of Sec. IV. We are grateful to Monica Guica and Ashoke Sen for comments on a draft of this paper. S. R. is partially supported by the Swarnajayanti fellowship of the Department of Science and Technology. S. R. would like to acknowledge the hospitality of the Kavli Institute for the Physics and Mathematics of the Universe (Tokyo), the Tata Institute of Fundamental Research (Mumbai), the National Institute for Science Education and Research (Bhubaneshwar), and the Institute of Theoretical Physics (Saclay) for hospitality while this work was being completed. We would also to acknowledge the Kavli Asian Winter School, ICTS/Prog-KAWS2018/01, for hospitality and thank all the participants of the Bangalore Area Strings Meeting, ICTS/Prog-basm2017/2017/07, for discussions.
[1] S. D. Mathur, The Fuzzball proposal for black holes: An elementary review, Fortschr. Phys. 53, 793 (2005); I. Bena and N. P. Warner, Black holes, black rings and their microstates, Lect. Notes Phys. 755, 1 (2008).

[2] I. Bena and N. P. Warner, Resolving the structure of black holes: Philosophizing with a Hammer, arXiv:1311.4538.

[3] V.S. Rychkov, D1-D5 black hole microstate counting from supergravity, J. High Energy Phys. 01 (2006) 063.
[4] O. Lunin and S. D. Mathur, Metric of the multiply wound rotating string, Nucl. Phys. B610, 49 (2001).

[5] O. Lunin, S. D. Mathur, and A. Saxena, What is the gravity dual of a chiral primary?, Nucl. Phys. B655, 185 (2003).

[6] S. D. Mathur, Fuzzballs and the information paradox: A summary and conjectures, arXiv:0810.4525.

[7] S. D. Mathur, The information paradox: A Pedagogical introduction, Classical Quantum Gravity 26, 224001 (2009). 
[8] S.D. Mathur, The information paradox: Conflicts and resolutions, Pramana 79, 1059 (2012).

[9] O. Lunin and S. D. Mathur, AdS/CFT duality and the black hole information paradox, Nucl. Phys. B623, 342 (2002).

[10] I. Bena, S. Giusto, E. J. Martinec, R. Russo, M. Shigemori, D. Turton, and N. P. Warner, Smooth Horizonless Geometries Deep Inside the Black-Hole Regime, Phys. Rev. Lett. 117, 201601 (2016).

[11] M. Cvetic and F. Larsen, Near horizon geometry of rotating black holes in five-dimensions, Nucl. Phys. B531, 239 (1998).

[12] S. Lloyd, Black holes, demons and the loss of coherence: How complex systems get information, and what they do with it, Ph.D. thesis, Rockefeller University, 1988.

[13] J. Kinney, J. M. Maldacena, S. Minwalla, and S. Raju, An index for 4 dimensional super conformal theories, Commun. Math. Phys. 275, 209 (2007).

[14] G. Casati, B. Chirikov, and I. Guarneri, Energy-Level Statistics of Integrable Quantum Systems, Phys. Rev. Lett. 54, 1350 (1985); M. V. Berry and M. Tabor, Level clustering in the regular spectrum, Proc. R. Soc. A 356, 375 (1977).

[15] I. Bena, C.-W. Wang, and N. P. Warner, Mergers and typical black hole microstates, J. High Energy Phys. 11 (2006) 042.

[16] A. Tyukov, R. Walker, and N. P. Warner, Tidal stresses and energy gaps in microstate geometries, J. High Energy Phys. 02 (2018) 122.

[17] I. Bena, P. Heidmann, and D. Turton, $\mathrm{AdS}_{2}$ holography: Mind the cap, J. High Energy Phys. 12 (2018) 028.

[18] M. Srednicki, Chaos and quantum thermalization, Phys. Rev. E 50, 888 (1994); The approach to thermal equilibrium in quantized chaotic systems, J. Phys. A 32, 1163 (1999).

[19] N. Lashkari, A. Dymarsky, and H. Liu, Eigenstate thermalization hypothesis in conformal field theory, J. Stat. Mech. (2018) 033101.

[20] S. D. Mathur and D. Turton, Comments on black holes I: The possibility of complementarity, J. High Energy Phys. 01 (2014) 034.

[21] S. El-Showk and K. Papadodimas, Emergent spacetime and holographic CFTs, J. High Energy Phys. 10 (2012) 106.

[22] A. Hamilton, D. N. Kabat, G. Lifschytz, and D. A. Lowe, Local bulk operators in AdS/CFT: A boundary view of horizons and locality, Phys. Rev. D 73, 086003 (2006); Holographic representation of local bulk operators, Phys. Rev. D 74, 066009 (2006); Local bulk operators in AdS/CFT: A holographic description of the black hole interior, Phys. Rev. D 75, 106001 (2007); Local bulk operators in AdS/CFT and the fate of the BTZ singularity, AMS/IP Stud. Adv. Math. 44, 85 (2008).

[23] P. Candelas, Vacuum polarization in Schwarzschild spacetime, Phys. Rev. D 21, 2185 (1980).

[24] A. Almheiri, D. Marolf, J. Polchinski, and J. Sully, Black holes: Complementarity or firewalls?, J. High Energy Phys. 02 (2013) 062.

[25] S. Ghosh and S. Raju, Loss of locality in gravitational correlators with a large number of insertions, Phys. Rev. D 96, 066033 (2017).

[26] D. N. Page, Average Entropy of a Subsystem, Phys. Rev. Lett. 71, 1291 (1993).

[27] S. Banerjee, J.-W. Bryan, K. Papadodimas, and S. Raju, A toy model of black hole complementarity, J. High Energy Phys. 05 (2016) 004.
[28] S. Ghosh and S. Raju, Breakdown of String Perturbation Theory for Many External Particles, Phys. Rev. Lett. 118, 131602 (2017).

[29] K. Papadodimas and S. Raju, An infalling observer in AdS/CFT, J. High Energy Phys. 10 (2013) 212.

[30] K. Skenderis and M. Taylor, The fuzzball proposal for black holes, Phys. Rep. 467, 117 (2008).

[31] E. T. Jaynes, Information theory and statistical mechanics, Phys. Rev. 106, 620 (1957); Information theory and statistical mechanics. ii, Phys. Rev. 108, 171 (1957).

[32] A. Almheiri, D. Marolf, J. Polchinski, D. Stanford, and J. Sully, An Apologia for firewalls, J. High Energy Phys. 09 (2013) 018; D. Marolf and J. Polchinski, Gauge/Gravity Duality and the Black Hole Interior, Phys. Rev. Lett. 111, 171301 (2013).

[33] K. Papadodimas and S. Raju, Black Hole Interior in the Holographic Correspondence and the Information Paradox, Phys. Rev. Lett. 112, 051301 (2014); State-dependent bulkboundary maps and black hole complementarity, Phys. Rev. D 89, 086010 (2014).

[34] K. Papadodimas and S. Raju, Local Operators in the Eternal Black Hole, Phys. Rev. Lett. 115, 211601 (2015); Remarks on the necessity and implications of state-dependence in the black hole interior, Phys. Rev. D 93, 084049 (2016).

[35] E. Verlinde and H. Verlinde, Passing through the Firewall, arXiv:1306.0515; Behind the horizon in AdS/CFT, arXiv:1311.1137; Black hole entanglement and quantum error correction, J. High Energy Phys. 10 (2013) 107; M. Guica and S. F. Ross, Behind the geon horizon, Classical Quantum Gravity 32, 055014 (2015).

[36] D. Harlow, Aspects of the Papadodimas-Raju proposal for the black hole interior, J. High Energy Phys. 11 (2014) 055; D. Marolf and J. Polchinski, Violations of the born rule in cool state-dependent horizons, J. High Energy Phys. 01 (2016) 008.

[37] S. Raju, Smooth causal patches for AdS black holes, Phys. Rev. D 95, 126002 (2017).

[38] D. L. Jafferis, Bulk reconstruction and the Hartle-Hawking wavefunction, arXiv:1703.01519.

[39] A. Bzowski, A. Gnecchi, and T. Hertog, Interactions resolve state-dependence in a toy-model of AdS black holes, J. High Energy Phys. 06 (2018) 167.

[40] A. Sen, Two charge system revisited: Small black holes or horizonless solutions?, J. High Energy Phys. 05 (2010) 097.

[41] F. Chen, B. Michel, J. Polchinski, and A. Puhm, Journey to the center of the Fuzzball, J. High Energy Phys. 02 (2015) 081.

[42] E. J. Martinec, S. Massai, and D. Turton, String dynamics in NS5-F1-P geometries, J. High Energy Phys. 09 (2018) 031.

[43] K. Skenderis and M. Taylor, Fuzzball Solutions and D1-D5 Microstates, Phys. Rev. Lett. 98, 071601 (2007); I. Kanitscheider, K. Skenderis, and M. Taylor, Holographic anatomy of fuzzballs, J. High Energy Phys. 04 (2007) 023; Fuzzballs with internal excitations, J. High Energy Phys. 06 (2007) 056.

[44] J. de Boer, K. Papadodimas, and E. Verlinde, Black Hole Berry Phase, Phys. Rev. Lett. 103, 131301 (2009).

[45] A. Bombini, A. Galliani, S. Giusto, E. Moscato, and R. Russo, Unitary 4-point correlators from classical geometries, Eur. Phys. J. C 78, 8 (2018); A. Galliani, S. Giusto, 
and R. Russo, Holographic 4-point correlators with heavy states, J. High Energy Phys. 10 (2017) 040; A. Galliani, S. Giusto, E. Moscato, and R. Russo, Correlators at large $\mathrm{c}$ without information loss, J. High Energy Phys. 09 (2016) 065.

[46] O. Lunin, J. M. Maldacena, and L. Maoz, Gravity solutions for the D1-D5 system with angular momentum, arXiv:hepth/0212210.

[47] C. Crnkovic and E. Witten, Covariant description of canonical formalism in geometrical theories, Three Hundred Years of Gravitation (Cambridge University Press, Cambridge, England, 1987), pp. 676-684; G. Zuckerman, Action principles and global geometry, Mathematical Aspects of String Theory (World Scientific, Singapore, 1987), Vol. 1, pp. 259-284.

[48] V. Balasubramanian, J. de Boer, S. El-Showk, and I. Messamah, Black holes as effective geometries, Classical Quantum Gravity 25, 214004 (2008).

[49] I. Bena, S. Giusto, R. Russo, M. Shigemori, and N. P. Warner, Habemus Superstratum: A constructive proof of the existence of superstrata, J. High Energy Phys. 05 (2015) 110.

[50] K. Skenderis and M. Taylor, Kaluza-Klein holography, J. High Energy Phys. 05 (2006) 057.

[51] I. Bena, D. Turton, R. Walker, and N. P. Warner, Integrability and black-hole microstate geometries, J. High Energy Phys. 11 (2017) 021.

[52] O. Aharony, S. S. Gubser, J. M. Maldacena, H. Ooguri, and Y. Oz, Large $\mathrm{N}$ field theories, string theory and gravity, Phys. Rep. 323, 183 (2000).

[53] J. C. Breckenridge, D. A. Lowe, R. C. Myers, A. W. Peet, A. Strominger, and C. Vafa, Macroscopic and microscopic entropy of near extremal spinning black holes, Phys. Lett. B 381, 423 (1996); J. C. Breckenridge, R. C. Myers, A. W. Peet, and C. Vafa, D-branes and spinning black holes, Phys. Lett. B 391, 93 (1997).

[54] M. Galassi, J. Davies, J. Theiler, B. Gough, G. Jungman, M. Booth et al., GNU Scientific Library Reference Manual (Network Theory, 2009), https://www.gnu.org/software/gsl/ manual/gsl-ref.pdf. 